\title{
Multiresolution Time-Domain Analysis of Multiconductor Transmission Lines Terminated in Linear Loads
}

\author{
Zongliang Tong, ${ }^{1}$ Lei Sun, ${ }^{1}$ Ying Li, ${ }^{1}$ Luis Diaz Angulo, ${ }^{2}$ \\ Salvador Gonzalez Garcia, ${ }^{2}$ and Jianshu Luo ${ }^{1}$ \\ ${ }^{1}$ College of Science, National University of Defense Technology, 137 Yanwachi Street, Changsha, Hunan 410073, China \\ ${ }^{2}$ Department of Electromagnetism and Matter Physics, University of Granada, Avda. Fuentenueva, s/n, 18071 Granada, Spain
}

Correspondence should be addressed to Jianshu Luo; jshluo@126.com

Received 22 October 2016; Revised 25 January 2017; Accepted 21 February 2017; Published 20 March 2017

Academic Editor: Michael Vynnycky

Copyright (C) 2017 Zongliang Tong et al. This is an open access article distributed under the Creative Commons Attribution License, which permits unrestricted use, distribution, and reproduction in any medium, provided the original work is properly cited.

\begin{abstract}
This paper derives a multiresolution time-domain (MRTD) scheme for the multiconductor transmission line (MTL) equations based on Daubechies' scaling functions. The terminations are characterized by a state-variable formulation which allows a general description of the termination networks. For the linear load terminations, a method incorporating the terminal constraints is proposed to work out the scheme at and close to the terminations. The MRTD scheme is implemented with different basis functions for linear components including resistances, inductances, and capacitances. Numerical results show that the MRTD schemes obtain a more stable result than the conventional finite difference time-domain (FDTD) method with a coarse space step.
\end{abstract}

\section{Introduction}

The multiconductor transmission line (MTL) equations have been thought to be an efficient model for interconnected lines [1]. With the increasing of the data transmission in electric systems, transient analysis of multiconductor transmission lines (MTLs) has significant meaning in circuit design and electromagnetic compatibility (EMC). For the problems in high frequency or with wide bandwidth, the time-domain algorithm can get the solution directly and analyze the integrated response and influence quickly. Several methods have been introduced to analyze the time-domain response, including the SPICE program, the time-domain to frequencydomain (TDFD) transformation method and the finite difference time-domain (FDTD) method [2]. Since the SPICE program can obtain the exact solution for the lossless lines, it has been tried to extend to the lossy case [3-6]. Some closed-form solutions for MTLs have been developed [7-10]. This method often needs convolution integrals to transform the solution from frequency-domain to time-domain. As a direct time-domain numerical method, the FDTD method has been used to solve the MTL equations naturally [11-13]. However, this method requires a fine spatial discretization along the lines for the rapidly varying signals, which leads to large memory requirements to guarantee accuracy.

For saving the memory of computing, we introduce the multiresolution time-domain (MRTD) scheme to solve the MTL equations in this paper. The MRTD scheme provides an efficient algorithm for the computation of electromagnetic fields because of its excellent capability to approximate exact solutions with lower sampling rates [14]. There are two kinds of MRTD schemes: one is to expand the variants by scaling functions in space domain, so it is called S-MRTD; the other one is to expand the variants by scaling functions and wavelet functions in space domain, so it is called WMRTD [14]. Since the Battle-Lemarie wavelet function used in [14] is not compactly supported, the iterative equations in MRTD need to be cut off and this may lead to truncation errors. To overcome this problem, some compactly supported scaling functions have been used to formulate the MRTD schemes [15-19]. To ensure the stability of the solutions and control dispersion errors of the different schemes, the stability condition and space sampling rules for these MRTD schemes have been studied in [20-24]. Furthermore, for MTL equations, the MRTD schemes can obtain an accurate solution with a coarse space interval. The MRTD scheme 
has been used to solve two-conductor transmission line equations with pure resistive loads [25], and we extend this method to the MTL equations terminated with linear load networks. The linear terminal networks can be described by state-variable characteristic and allow us to model the terminal constraints directly $[26,27]$.

In this paper, we use MRTD scheme to analyze the terminal response of MTLs with linear loads. In Section 2, the MRTD scheme is derived based on Daubechies' scaling functions for the lossy MTL equations. In Section 3, the iterative equations for terminal voltages are derived based on the linear terminal networks. The iterative equations of voltages and currents close to the terminals are modified. In Section 4, some numerical results are presented for the terminal response of both purely resistive terminations and the terminations network including inductances and capacitances. The numerical results are compared to the results obtained with SPICE and FDTD methods.

\section{MRTD for MTL Equations}

In this section, we derive the S-MRTD scheme for MTL equations. Similarly to the FDTD method, the expansions of the currents are shifted by half a discretization interval in space and time with respect to the expansions of the voltages.

The homogeneous equations for $(m+1)$-conductor lines can be written as

$$
\begin{gathered}
\frac{\partial}{\partial z} \mathbf{V}(z, t)+\mathbf{R I}(z, t)+\mathbf{L} \frac{\partial}{\partial t} \mathbf{I}(z, t)=0 \\
\frac{\partial}{\partial z} \mathbf{I}(z, t)+\mathbf{G V}(z, t)+\mathbf{C} \frac{\partial}{\partial t} \mathbf{V}(z, t)=0
\end{gathered}
$$

where the column vectors $\mathbf{V}(z, t)$ and $\mathbf{I}(z, t)$ represent the voltages and currents along the $m$ conductors (the other one is the reference conductor) and contain $m$ voltages $V_{i}(z, t)$ and $m$ currents $I_{i}(z, t)$ as shown below:

$$
\begin{aligned}
& \mathbf{V}(z, t)=\left[V_{1}(z, t), \ldots, V_{i}(z, t), \ldots, V_{m}(z, t)\right]^{T} \\
& \mathbf{I}(z, t)=\left[I_{1}(z, t), \ldots, I_{i}(z, t), \ldots, I_{m}(z, t)\right]^{T} .
\end{aligned}
$$

$m \times m$ matrices $\mathbf{R}, \mathbf{L}, \mathbf{G}$, and $\mathbf{C}$ represent the per-unitlength resistance, inductance, conductance, and capacitance, respectively.

The voltage vector $\mathrm{V}(z, t)$ and current vector $\mathbf{I}(z, t)$ can be expanded as follows:

$$
\begin{aligned}
& \mathbf{V}(z, t)=\sum_{k, n=-\infty}^{+\infty} \mathbf{V}_{k}^{n} \varphi_{k}(z) h_{n}(t) \\
& \mathbf{I}(z, t)=\sum_{k, n=-\infty}^{+\infty} \mathbf{I}_{k+1 / 2}^{n+1 / 2} \varphi_{k+1 / 2}(z) h_{n+1 / 2}(t),
\end{aligned}
$$

where $\mathbf{V}_{k}^{n}$ and $\mathbf{I}_{k+1 / 2}^{n+1 / 2}$ are the coefficient vectors for the voltages and currents in terms of scaling functions, respectively. $\mathbf{V}_{k}^{n}$
TABLE 1: Connection coefficients $a(i)$ of Daubechies' scaling functions.

\begin{tabular}{lccc}
\hline$i$ & $\mathrm{Db} 2$ & $\mathrm{Db3}$ & $\mathrm{Db} 4$ \\
\hline 0 & 1.2291666667 & 1.2918129281 & 1.3110340773 \\
1 & -0.0937500000 & -0.1371343465 & -0.1560100110 \\
2 & 0.0104166667 & 0.0287617728 & 0.0419957460 \\
3 & & -0.0034701413 & -0.0086543236 \\
4 & & 0.0000080265 & 0.0008308695 \\
5 & & & 0.0000108999 \\
6 & & & -0.0000000041 \\
\hline$q_{\max }$ & 0.7500 & 0.6844 & 0.6585 \\
\hline
\end{tabular}

and $\mathbf{I}_{k+1 / 2}^{n+1 / 2}$ are $m$ dimensions vectors which are represented as follows:

$$
\begin{aligned}
\mathbf{V}_{k}^{n} & =\left[V_{1, k}^{n}, \ldots, V_{i, k}^{n}, \ldots, V_{m, k}^{n}\right]^{T}, \\
\mathbf{I}_{k+1 / 2}^{n+1 / 2} & =\left[I_{1, k+1 / 2}^{n+1 / 2}, \ldots, I_{i, k+1 / 2}^{n+1 / 2}, \ldots, I_{m, k+1 / 2}^{n+1 / 2}\right]^{T} .
\end{aligned}
$$

The index $i$ represents the number of the conductors and the indices $n$ and $k$ are the discrete spatial and temporal index with respect to space and time coordinates via $z=k \Delta z$ and $t=n \Delta t$, where $\Delta z$ and $\Delta t$ represent the spatial and temporal discretization intervals in $z$ and $t$ direction. The functions $h_{n}(t)$ and $\varphi_{k}(z)$ are defined as

$$
\begin{aligned}
h_{n}(t) & =h\left(\frac{t}{\Delta t}-n\right), \\
\varphi_{k}(z) & =\varphi\left(\frac{z}{\Delta z}-k\right) .
\end{aligned}
$$

The function $h(t)$ is defined as Haar's scaling function and $\varphi(z)$ is Daubechies' scaling function.

To derive the MRTD scheme, we need the following formulations:

$$
\begin{gathered}
\left\langle h_{n}(t), h_{n^{\prime}}(t)\right\rangle=\delta_{n, n^{\prime}} \Delta t \\
\left\langle h_{n}(t), \frac{\partial h_{n^{\prime}+1 / 2}(t)}{\partial t}\right\rangle=\delta_{n, n^{\prime}}-\delta_{n, n^{\prime}+1}
\end{gathered}
$$

where $\delta_{n, n^{\prime}}$ represents the Kronecker symbol.

$$
\begin{gathered}
\left\langle\varphi_{k}(z), \varphi_{k^{\prime}}(z)\right\rangle=\delta_{k, k^{\prime}} \Delta z \\
\left\langle\varphi_{k}(z), \frac{\partial \varphi_{k^{\prime}+1 / 2}(z)}{\partial z}\right\rangle=\sum_{i=-L_{S}}^{L_{S}-1} a(i) \delta_{k+i, k^{\prime}}
\end{gathered}
$$

where $L_{S}$ denotes the effective support size of the basis functions. The coefficients $a(i)$ are called connection coefficients and Table 1 lists $a(i)$ for $0 \leq i \leq L_{S}-1$ according to [22]. For $i>L_{S}-1, a(i)=0$, and, for $i<0, a(i)$ fulfills the symmetry relation $a(-1-i)=-a(i)$.

Daubechies' scaling functions satisfy the shifted interpolation property [28]

$$
\varphi\left(i+M_{1}\right)=\delta_{i, 0}
$$


for $i$ integer, where $M_{1}=\int_{-\infty}^{+\infty} z \phi(z) d z$ is the first-moment of the scaling functions. [16]

Making use of (9), the basis function (6) is modified as

$$
\varphi_{k}(z)=\varphi\left(\frac{z}{\Delta z}-k+M_{1}\right)
$$

In spite of the support set length of the scaling functions [29], single point sampling of the total voltages and currents can be taken at integer points with negligible error. Taking voltage at spatial point $k \Delta z$ and at time $n \Delta t$, for example, we obtain

$$
\begin{aligned}
& \mathbf{V}(k \Delta z, n \Delta t)=\iint_{-\infty}^{+\infty} \sum_{k^{\prime}, n^{\prime}=-\infty}^{+\infty} \mathbf{V}_{k^{\prime}}^{n^{\prime}} \varphi_{k^{\prime}}(z) h_{n^{\prime}}(t) \\
& \cdot \delta\left(\frac{z}{\Delta z}-k\right) \delta\left(\frac{t}{\Delta t}-n\right) d z d t=\sum_{k^{\prime}, n^{\prime}=-\infty}^{+\infty} \mathbf{V}_{k^{\prime}}^{n^{\prime}} \varphi(k \\
& \left.-k^{\prime}+M_{1}\right) h\left(n-n^{\prime}\right)=\mathbf{V}_{k}^{n},
\end{aligned}
$$

where $\delta$ is the Dirac delta function. Equation (11) means the voltage value at each integer point is equal to the expansion coefficient. The current values have the same character at each half-integer point. Therefore, we use $\mathbf{V}_{k}^{n}$ and $\mathbf{I}_{k+1 / 2}^{n+1 / 2}$ directly to represent the voltages at $(k \Delta z, n \Delta t)$ and the currents at $((k+$ $1 / 2) \Delta z,(n+1 / 2) \Delta t)$ in this paper.

The modified $\varphi_{k}(z)$ in (10) also satisfies integrals (8). Substituting (3a) and (3b) into (1a) and (1b) and applying the Galerkin method to (1a) and (1b), which means using $h_{n}(t) \varphi_{k+1 / 2}(z)$ as the test function for (1a) and $h_{n+1 / 2}(t) \varphi_{k}(z)$ as the test function for (1b), we can obtain the following iterative equations for the voltages and currents:

$$
\begin{aligned}
& \mathbf{V}_{k}^{n+1} \\
& \quad=\mathbf{A}_{1}\left[\mathbf{A}_{2} \mathbf{V}_{k}^{n}-\frac{\Delta t}{\Delta z} \sum_{i=0}^{L_{S}-1} a(i)\left(\mathbf{I}_{k+i+1 / 2}^{n+1 / 2}-\mathbf{I}_{k-i-1 / 2}^{n+1 / 2}\right)\right], \\
& \mathbf{I}_{k+1 / 2}^{n+1 / 2} \\
& \quad=\mathbf{B}_{1}\left[\mathbf{B}_{2} \mathbf{I}_{k+1 / 2}^{n-1 / 2}-\frac{\Delta t}{\Delta z} \sum_{i=0}^{L_{S}-1} a(i)\left(\mathbf{V}_{k+i+1}^{n}-\mathbf{V}_{k-i}^{n}\right)\right],
\end{aligned}
$$

where $a(i)$ is the connection coefficient and $\mathbf{A}_{1}, \mathbf{A}_{2}, \mathbf{B}_{1}$, and $\mathbf{B}_{2}$ are constant matrices:

$$
\begin{aligned}
& \mathbf{A}_{1}=\left(\mathbf{C}+\frac{\Delta t}{2} \mathbf{G}\right)^{-1}, \\
& \mathbf{A}_{2}=\left(\mathbf{C}-\frac{\Delta t}{2} \mathbf{G}\right), \\
& \mathbf{B}_{1}=\left(\mathbf{L}+\frac{\Delta t}{2} \mathbf{R}\right)^{-1}, \\
& \mathbf{B}_{2}=\left(\mathbf{L}-\frac{\Delta t}{2} \mathbf{R}\right) .
\end{aligned}
$$

For the stability of the solution, the temporal and spatial discretization should satisfy the Courant condition [20,25]

$$
\frac{v_{\max } \Delta t}{\Delta z} \leq \frac{1}{\sum_{i=0}^{L_{S^{-}}}|a(i)|},
$$

where $v_{\max }$ represents the maximum propagation velocity of the modes. For MTLs in inhomogeneous media that have $n$ velocities of propagation of the modes, the Courant condition has to be satisfied by the largest of the mode velocities, and thereby it will be satisfied by the smaller mode velocities.

Note the Courant number as

$$
q=\frac{v \Delta t}{\Delta z}
$$

The maximum values of $q$ required by a stable algorithm can be calculated from the connection coefficients. Table 1 lists $q_{\max }$ for different Daubechies' scaling functions. For one-dimensional problems, the stability condition for the conventional FDTD method can be written as [30]

$$
\frac{v_{\max } \Delta t}{\Delta z} \leq 1
$$

Inequality (16) indicates that, for the conventional FDTD method, the maximum Courant number to guarantee the stability of the differential scheme is $q_{\max }=1$. In Table 1 , all $q_{\max }$ values for MRTD schemes are smaller than 1 , which means the MRTD schemes must obey a stricter stability condition compared to the conventional FDTD method. When using the same space step for MRTD schemes and FDTD method, the MRTD schemes need a smaller time step to guarantee the stability of the algorithm.

\section{Terminal Formulations for Linear Loads}

In this section, we consider the iterative equations at the source and the load. Since the iterative equations in (12a) and (12b) are not suitable for the terminations, we need to derive the iterative equations with the terminal conditions. As shown in Figure 1, we assume the length of the lines to be $L$, the lines are divided uniformly into NDZ segments with the space interval $\Delta z$, and the total solution time is divided into NDT steps with the uniform time interval $\Delta t$. Since we have expanded the voltages $\mathbf{V}(z, t)$ at the integer points and currents $\mathbf{I}(z, t)$ at the half-integer points in (3a) and (3b), the voltages and currents will appear alternatively along the lines as shown in Figure 1.

As the iterative equations (12a) and (12b) indicate, there are two parts in the iterative equations which we need to update. One part is the voltage iterative equations at the terminals. Since all the currents are located at the inner points along the lines, we do not need to analyze the currents at the terminals. The other part is the voltages and currents near the terminals in which the voltages are $\mathbf{V}_{i}^{n}$ and $\mathbf{V}_{\mathrm{NDZ}-i}^{n}$, for $i=1,2, \ldots, L_{S}-1$, and the currents are $\mathbf{I}_{i+1 / 2}^{n+1 / 2}$ and $\mathbf{I}_{\mathrm{NDZ}-i+1 / 2}^{n+1 / 2}$ for $i=0,1, \ldots, L_{S}-2$. The iterative equations of these voltages and currents contain some terms exceeding the index range.

Taking voltages, for example, to derive the modified iterative equations, the modified iterative equations for currents 


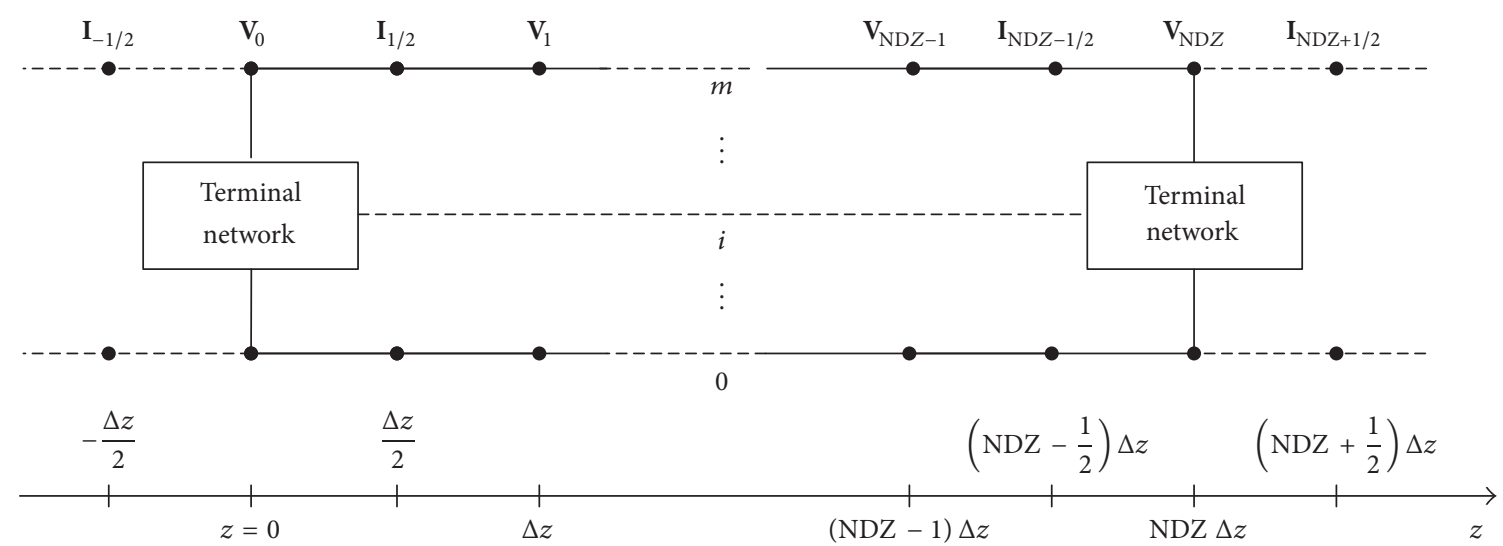

FIGURE 1: Discretization of the voltages and currents.

can be obtained by the similar steps. Using the relation of the connection coefficients [17]

$$
\sum_{i=0}^{L_{S}-1}(2 i+1) a(i)=1
$$

substituting (17) into (12a) gives

$$
\begin{aligned}
& \sum_{i=0}^{L_{S}-1}(2 i+1) a(i) \mathbf{V}_{k}^{n+1}=\mathbf{A}_{1}\left[\sum_{i=0}^{L_{S}-1}(2 i+1) a(i) \mathbf{A}_{2} \mathbf{V}_{k}^{n+1}\right. \\
& \left.\quad+\sum_{i=0}^{L_{S}-1} \frac{\Delta t}{(2 i+1) \Delta z} a(i)(2 i+1)\left(\mathbf{I}_{k+i+1 / 2}^{n+1 / 2}-\mathbf{I}_{k-i-1 / 2}^{n+1 / 2}\right)\right] .
\end{aligned}
$$

Considering the corresponding terms with $i$, we can decompose (18) as [18]

$$
\begin{aligned}
& a(0) \mathbf{V}_{k}^{n+1}=\mathbf{A}_{1}\left[a(0) \mathbf{A}_{2} \mathbf{V}_{k}^{n}-a(0)\right. \\
& \left.\cdot \frac{\Delta t}{\Delta z}\left(\mathbf{I}_{k+1 / 2}^{n+1 / 2}-\mathbf{I}_{k-1 / 2}^{n+1 / 2}\right)\right] \\
& 3 a(1) \mathbf{V}_{k}^{n+1}=\mathbf{A}_{1}\left[3 a(1) \mathbf{A}_{2} \mathbf{V}_{k}^{n}-3 a(1)\right. \\
& \left.\cdot \frac{\Delta t}{3 \Delta z}\left(\mathbf{I}_{k+3 / 2}^{n+1 / 2}-\mathbf{I}_{k-3 / 2}^{n+1 / 2}\right)\right]
\end{aligned}
$$

$$
\begin{aligned}
& (2 i+1) a(i) \mathbf{V}_{k}^{n+1}=\mathbf{A}_{1}\left[(2 i+1) a(i) \mathbf{A}_{2} \mathbf{V}_{k}^{n+1}\right. \\
& \left.-(2 i+1) a(i) \frac{\Delta t}{(2 i+1) \Delta z}\left(\mathbf{I}_{k+i+1 / 2}^{n+1 / 2}-\mathbf{I}_{k-i-1 / 2}^{n+1 / 2}\right)\right]
\end{aligned}
$$

$$
\left(2 L_{S}-1\right) a\left(L_{S}-1\right) \mathbf{V}_{k}^{n+1}=\mathbf{A}_{1}\left[\left(2 L_{S}-1\right)\right.
$$

$$
\begin{aligned}
& \cdot a\left(L_{S}-1\right) \mathbf{A}_{2} \mathbf{V}_{k}^{n+1}-\left(2 L_{S}-1\right) a\left(L_{S}-1\right) \\
& \left.\cdot \frac{\Delta t}{\left(2 L_{S}-1\right) \Delta z}\left(\mathbf{I}_{k+L_{S}-1 / 2}^{n+1 / 2}-\mathbf{I}_{k-L_{S}+1 / 2}^{n+1 / 2}\right)\right]
\end{aligned}
$$

for $i=0,1, \ldots, L_{S}-1$.

Equation (19c) indicates that each subequation in (19a), (19b), (19c), and (19d) is a central difference scheme of the FDTD method with the spatial interval $(2 i+1) \Delta z$. Summing up all the subequations in (19a), (19b), (19c), and (19d) we can obtain (18), so we can view the MRTD schemes as the weighted mean of the conventional FDTD method, and the weighting number is $(2 i+1) a(i)$.

By viewing the MRTD schemes as the weighted mean of the conventional FDTD method, (18) also indicate that the MRTD schemes contain $L_{S}$ differential terms in each iterative equation. For the conventional FDTD method, the iterative equations only contain 1 differential term for onedimensional problems. Ignoring the addition terms in the iterative equations, that means the computational complexity of MRTD is $L_{S}$ times of conventional FDTD under the same grid numbers.

We will use this relationship between FDTD method and MRTD scheme to derive the voltage iterative equations at the terminals and the voltages and currents close to the terminals. Firstly, we take $\mathbf{V}_{0}^{n+1}$ as an example to derive the voltage iterative equations at the terminals. 
Decomposing $\mathbf{V}_{0}^{n+1}$ as (19a), (19b), (19c), and (19d), we can obtain each subequation as follows:

$$
\begin{aligned}
& (2 i+1) a(i) \mathbf{V}_{0}^{n+1}=\mathbf{A}_{1}\left[(2 i+1) a(i) \mathbf{A}_{2} \mathbf{V}_{0}^{n}\right. \\
& \left.-(2 i+1) a(i) \frac{\Delta t}{(2 i+1) \Delta z}\left(\mathbf{I}_{i+1 / 2}^{n+1 / 2}-\mathbf{I}_{-i-1 / 2}^{n+1 / 2}\right)\right]
\end{aligned}
$$

for $i=0,1, \ldots, L_{S}-1$.

As shown in Figure 1 , the term $\mathbf{I}_{-i-1 / 2}^{n+1 / 2}\left(\right.$ for $i=0,1, \ldots, L_{S}-$ 1) in (20) does not exist, its subscript for spatial discretization exceeds the index range, and therefore the central difference scheme is not suitable for (20). Using the forward difference scheme to replace the central difference scheme, we obtain

$$
\begin{aligned}
& (2 i+1) a(i) \mathbf{V}_{0}^{n+1}=\mathbf{A}_{1}\left[(2 i+1) a(i) \mathbf{A}_{2} \mathbf{V}_{0}^{n}\right. \\
& \left.-(2 i+1) a(i) \frac{\Delta t}{(2 i+1) \Delta z / 2}\left(\mathbf{I}_{i+1 / 2}^{n+1 / 2}-\mathbf{I}_{0}^{n+1 / 2}\right)\right]
\end{aligned}
$$

for $i=0,1, \ldots, L_{S}-1$.

Summing up all the subequations in (21), we obtain the iterative equations at the terminals

$$
\mathbf{V}_{0}^{n+1}=\mathbf{A}_{1}\left[\mathbf{A}_{2} \mathbf{V}_{0}^{n}-2 \frac{\Delta t}{\Delta z} \sum_{i=0}^{L_{S}-1} a(i)\left(\mathbf{I}_{i+1 / 2}^{n+1 / 2}-\mathbf{I}_{0}^{n+1 / 2}\right)\right]
$$

In (22), the term $\mathbf{I}_{0}^{n+1 / 2}$ represents the current expansion coefficient at the point $0 \Delta z$, and since the terminal network is located at the point $0 \Delta z$, the formulation of $\mathbf{I}_{0}^{n+1 / 2}$ must satisfy the constraint condition from the terminal network.

In the case of a linear network, the terminal can be characterized in a state-variable form [13]

$$
\frac{d}{d t} \mathbf{X}(t)=\mathbf{M X}(t)+\mathbf{N U}(t)
$$

with an associated output relation

$$
\mathbf{Y}(t)=\mathbf{O X}(t)+\mathbf{P} \mathbf{U}(t)+\mathbf{Q} \frac{d}{d t} \mathbf{U}(t)
$$

The vector $\mathbf{X}$ contains the state variables of the lumped network. These are typically the inductor currents and capacitor voltages in that network or some subset of those variables. The vector $\mathbf{U}$ contains the independent sources in the network (the inputs), and the vector $\mathbf{Y}$ contains the designated outputs (currents and/or voltages) of the network. $\mathbf{M}, \mathbf{N}, \mathbf{O}, \mathbf{P}$, and $\mathbf{Q}$ are constant coefficient matrices, which are all determined by the specific linear network.
Define the output in (23a) and (23b) as $\mathbf{I}_{S}$ and $\mathbf{I}_{L}$, and then

$$
\begin{aligned}
\frac{d}{d t} \mathbf{X}_{S}(t)= & \mathbf{M}_{S} \mathbf{X}_{S}(t)+\mathbf{N}_{0} \mathbf{V}_{0}(t)+\mathbf{N}_{S} \mathbf{S}_{S}(t) \\
\mathbf{I}_{S}(t)= & \mathbf{O}_{S} \mathbf{X}_{S}(t)+\mathbf{P}_{0} \mathbf{V}_{0}(t)+\mathbf{P}_{S}(t) \mathbf{S}_{S}(t) \\
& +\mathbf{Q}_{0} \frac{d}{d t} \mathbf{V}_{0}(t)+\mathbf{Q}_{S} \frac{d}{d t} \mathbf{S}_{S}(t) \\
\frac{d}{d t} \mathbf{X}_{L}(t)= & \mathbf{M}_{L} \mathbf{X}_{L}(t)+\mathbf{N}_{\mathrm{NDZ}} \mathbf{V}_{\mathrm{NDZ}}(t)+\mathbf{N}_{L} \mathbf{S}_{L}(t), \\
\mathbf{I}_{L}(t)= & \mathbf{O}_{L} \mathbf{X}_{L}(t)+\mathbf{P}_{\mathrm{NDZ}} \mathbf{V}_{\mathrm{NDZ}}(t)+\mathbf{P}_{L}(t) \mathbf{S}_{L}(t) \\
& +\mathbf{Q}_{\mathrm{NDZ}} \frac{d}{d t} \mathbf{V}_{\mathrm{NDZ}}(t)+\mathbf{Q}_{L} \frac{d}{d t} \mathbf{S}_{L}(t),
\end{aligned}
$$

where $S$ and $L$ represent the networks at the source and the load.

Since the variables $\mathbf{I}_{S}(t), \mathbf{X}_{S}(t)$, and $\mathbf{S}_{S}(t)$ are located at the integer point, we can expand them as (3a) and (3b):

$$
\begin{aligned}
& \mathbf{I}_{S}(t)=\sum_{n=-\infty}^{+\infty} \mathbf{I}_{S}^{n+1 / 2} h_{n+1 / 2}(t), \\
& \mathbf{X}_{S}(t)=\sum_{n=-\infty}^{+\infty} \mathbf{X}_{S}^{n+1 / 2} h_{n+1 / 2}(t), \\
& \mathbf{S}_{S}(t)=\sum_{n=-\infty}^{+\infty} \mathbf{S}_{S}^{n} h_{n}(t) .
\end{aligned}
$$

Here, we expand $\mathbf{I}_{S}(t)$ and $\mathbf{X}_{S}(t)$ at the half-integer point to correspond with the current $\mathbf{I}_{0}^{n+1 / 2}$ and this will lead to an explicit expression for the iterative equations below. $\mathbf{V}_{0}(t)$ is also needed in (24a) and (24b), space sampling the voltage at the point $0 \Delta z$, and we can obtain

$$
\mathbf{V}_{0}(t)=\left\langle\mathbf{V}(z, t), \varphi_{0}(z)\right\rangle=\sum_{n=-\infty}^{+\infty} \mathbf{V}_{0}^{n} h_{n}(t)
$$

Substituting (26)-(27) into (24a) and (24b), taking $h_{n}(t)$ as the test function for $(24 \mathrm{a})$ and $h_{n+1 / 2}(t)$ as the test function for (24b), and applying the Galerkin method to (24a) and (24b), we obtain

$$
\begin{aligned}
\frac{\mathbf{X}_{S}^{n+1 / 2}-\mathbf{X}_{S}^{n-1 / 2}}{\Delta t}= & \mathbf{M}_{S} \frac{\mathbf{X}_{S}^{n+1 / 2}+\mathbf{X}_{S}^{n-1 / 2}}{2}+\mathbf{N}_{0} \mathbf{V}_{0}^{n} \\
& +\mathbf{N}_{S} \mathbf{S}_{S}^{n}, \\
\mathbf{I}_{S}^{n+1 / 2}= & \mathbf{O}_{S} \mathbf{X}_{S}^{n+1 / 2}+\mathbf{P}_{0} \frac{\mathbf{V}_{0}^{n+1}+\mathbf{V}_{0}^{n}}{2} \\
& +\mathbf{P}_{S} \frac{\mathbf{V}_{S}^{n+1}+\mathbf{V}_{S}^{n}}{2} \\
& +\mathbf{Q}_{0} \frac{\mathbf{V}_{0}^{n+1}-\mathbf{V}_{0}^{n}}{\Delta t} \\
& +\mathbf{Q}_{S} \frac{\mathbf{S}_{S}^{n+1}-\mathbf{S}_{S}^{n}}{\Delta t}
\end{aligned}
$$


Substituting (28b) into (22)

$$
\begin{gathered}
\mathbf{V}_{0}^{n+1}=\mathbf{A}_{3}\left\{\mathbf{A}_{4} \mathbf{V}_{0}^{n}-\sum_{i=0}^{L_{S}-1} 2 a(i) \mathbf{I}_{i+1 / 2}^{n+1 / 2}+\sum_{i=0}^{L_{S}-1} 2 a(i)\right. \\
\cdot \mathbf{O}_{S} \mathbf{X}_{S}^{n+1 / 2}+\sum_{i=0}^{L_{S}-1} a(i) \\
\left.\cdot\left[\mathbf{P}_{S}\left(\mathbf{V}_{S}^{n+1}+\mathbf{V}_{S}^{n}\right)+\frac{2}{\Delta t} \mathbf{Q}_{S}\left(\mathbf{S}_{S}^{n+1}-\mathbf{S}_{S}^{n}\right)\right]\right\},
\end{gathered}
$$

where

$$
\begin{aligned}
& \mathbf{A}_{3}= {\left[\frac{\Delta z}{\Delta t} \mathbf{C}+\frac{\Delta z}{2} \mathbf{G}+\sum_{i=0}^{L_{S}-1} a(i)\left(\mathbf{P}_{0}+\frac{2}{\Delta t} \mathbf{Q}_{0}\right)\right]^{-1}, } \\
& \mathbf{A}_{4}= {\left[\frac{\Delta z}{\Delta t} \mathbf{C}-\frac{\Delta z}{2} \mathbf{G}-\sum_{i=0}^{L_{S}-1} a(i)\left(\mathbf{P}_{0}-\frac{2}{\Delta t} \mathbf{Q}_{0}\right)\right], } \\
& \mathbf{X}_{S}^{n+1 / 2}=\left(\mathbf{1}_{m}-\frac{\Delta t}{2} \mathbf{M}_{S}\right)^{-1} \\
& .\left[\left(\mathbf{1}_{m}+\frac{\Delta t}{2} \mathbf{M}_{S}\right)+\Delta t\left(\mathbf{N}_{0} \mathbf{V}_{0}^{n}+\mathbf{N}_{S} \mathbf{S}_{S}^{n}\right)\right],
\end{aligned}
$$

where $\mathbf{1}_{m}$ represents $m$ dimension unity matrix.

Note that the expression of $\mathbf{X}_{S}$ in (32) is a little different from the iterative equations in $[2,13]$. The expression of state variable $\mathbf{X}_{S}^{n+1 / 2}$ in this paper falls behind the terminal voltage $\mathbf{V}_{0}^{n+1}$ half a temporal step, while in $[2,13]$ the state variable and the terminal voltage are at the same time point. So the state variable and the terminal voltage are coupling in $[2,13]$, and there is a matrix inversion process to obtain the state variables and the terminal voltages. On the other hand, these two variables are decoupled in (28a) and (28b). That means we separate the iterative equations of the state variables and the terminal voltages, and this separation reduces the matrix inversion process to obtain the state variables and the terminal voltages.

Following a similar procedure, we obtain

$$
\begin{aligned}
\mathbf{V}_{\mathrm{NDZ}}^{n+1}=\mathbf{A}_{5}\left\{\mathbf{A}_{6} \mathbf{V}_{\mathrm{NDZ}}^{n}+\sum_{i=0}^{L_{S}-1} 2 a(i) \mathbf{I}_{\mathrm{NDZ}-i-1 / 2}^{n+1 / 2}\right. \\
-\sum_{i=0}^{L_{S}-1} 2 a(i) \mathbf{O}_{L} \mathbf{X}_{L}^{n+1 / 2}-\sum_{i=0}^{L_{S}-1} a(i) \\
\left.\cdot\left[\mathbf{P}_{L}\left(\mathbf{V}_{L}^{n+1}+\mathbf{V}_{L}^{n}\right)+\frac{2}{\Delta t} \mathbf{Q}_{L}\left(\mathbf{S}_{L}^{n+1}-\mathbf{S}_{L}^{n}\right)\right]\right\},
\end{aligned}
$$

where

$$
\begin{aligned}
& \mathbf{A}_{5}= {\left[\frac{\Delta z}{\Delta t} \mathbf{C}+\frac{\Delta z}{2} \mathbf{G}\right.} \\
&\left.+\sum_{i=0}^{L_{S}-1} a(i)\left(\mathbf{P}_{\mathrm{NDZ}}+\frac{2}{\Delta t} \mathbf{Q}_{\mathrm{NDZ}}\right)\right]^{-1}, \\
& \mathbf{A}_{6}= {\left[\frac{\Delta z}{\Delta t} \mathbf{C}-\frac{\Delta z}{2} \mathbf{G}-\sum_{i=0}^{L_{S}-1} a(i)\left(\mathbf{P}_{\mathrm{NDZ}}-\frac{2}{\Delta t} \mathbf{Q}_{\mathrm{NDZ}}\right)\right], } \\
& \mathbf{X}_{L}^{n+1 / 2}=\left(\mathbf{1}_{m}-\frac{\Delta t}{2} \mathbf{M}_{L}\right)^{-1}\left[\left(\mathbf{1}_{m}+\frac{\Delta t}{2} \mathbf{M}_{L}\right)\right. \\
&\left.+\Delta t\left(\mathbf{N}_{\mathrm{NDZ}} \mathbf{V}_{\mathrm{NDZ}}^{n}+\mathbf{N}_{L} \mathbf{S}_{L}^{n}\right)\right] .
\end{aligned}
$$

In the case of resistance terminations, the generalized Thevenin equivalent representation in (24a) and (24b) is written as $[2,13]$

$$
\begin{aligned}
& \mathbf{I}_{S}=-\mathbf{G}_{S} \mathbf{V}_{0}+\mathbf{G}_{S} \mathbf{V}_{S}, \\
& \mathbf{I}_{L}=\mathbf{G}_{L} \mathbf{V}_{\mathrm{NDZ}}+\mathbf{G}_{L} \mathbf{V}_{L},
\end{aligned}
$$

where $\mathbf{G}_{S}=\mathbf{R}_{S}^{-1}$ and $\mathbf{G}_{L}=\mathbf{R}_{L}^{-1}$.

Hence, $\mathbf{X}_{S}=\mathbf{X}_{L}=0, \mathbf{M}_{S}=\mathbf{N}_{0}=\mathbf{N}_{S}=\mathbf{O}_{S}=\mathbf{Q}_{0}=\mathbf{Q}_{S}=$ $\mathbf{0}, \mathbf{M}_{L}=\mathbf{N}_{\mathrm{NDZ}}=\mathbf{N}_{L}=\mathbf{O}_{L}=\mathbf{Q}_{\mathrm{NDZ}}=\mathbf{Q}_{L}=\mathbf{0}, \mathbf{P}_{0}=\mathbf{G}_{S}$, $\mathbf{P}_{S}=\mathbf{G}_{S}, \mathbf{P}_{\mathrm{NDZ}}=\mathbf{G}_{L}$, and $\mathbf{P}_{L}=-\mathbf{G}_{L}$.

The iterative equations of the terminal voltages can be reduced as

$$
\begin{aligned}
& \mathbf{V}_{0}^{n+1}=\mathbf{A}_{7}\left[\mathbf{A}_{8} \mathbf{V}_{0}^{n}-2 \mathbf{R}_{S} \sum_{i=0}^{L_{S}-1} a(i) \mathbf{I}_{1 / 2}^{n+1 / 2}\right. \\
& \left.+\left(\mathbf{V}_{S}^{n+1}+\mathbf{V}_{S}^{n}\right)\right], \\
& \mathbf{V}_{\mathrm{NDZ}}^{n+1}=\mathbf{A}_{9}\left[\mathbf{A}_{10} \mathbf{V}_{\mathrm{NDZ}}^{n}+2 \mathbf{R}_{L} \sum_{i=0}^{L_{S}-1} a(i) \mathbf{I}_{\mathrm{NDZ}-1 / 2}^{n+1 / 2}\right. \\
& \left.+\left(\mathbf{V}_{L}^{n+1}+\mathbf{V}_{L}^{n}\right)\right]
\end{aligned}
$$

where

$$
\begin{aligned}
& \mathbf{A}_{7}=\left[\frac{\Delta z}{\Delta t} \mathbf{R}_{S} \mathbf{C}+\frac{\Delta z}{2} \mathbf{R}_{S} \mathbf{G}+\sum_{i=0}^{L_{S}-1} a(i) \mathbf{1}_{m}\right]^{-1}, \\
& \mathbf{A}_{8}=\left[\frac{\Delta z}{\Delta t} \mathbf{R}_{S} \mathbf{C}-\frac{\Delta z}{2} \mathbf{R}_{S} \mathbf{G}-\sum_{i=0}^{L_{S}-1} a(i) \mathbf{1}_{m}\right], \\
& \mathbf{A}_{9}=\left[\frac{\Delta z}{\Delta t} \mathbf{R}_{L} \mathbf{C}+\frac{\Delta z}{2} \mathbf{R}_{L} \mathbf{G}+\sum_{i=0}^{L_{S}-1} a(i) \mathbf{1}_{m}\right]^{-1}, \\
& \mathbf{A}_{10}=\left[\frac{\Delta z}{\Delta t} \mathbf{R}_{L} \mathbf{C}-\frac{\Delta z}{2} \mathbf{R}_{L} \mathbf{G}-\sum_{i=0}^{L_{S}-1} a(i) \mathbf{1}_{m}\right] .
\end{aligned}
$$


For the iterative equations of voltages and currents near the terminals, we will use the truncation method to remove the terms whose index exceeds the range. This method is based on the point to view the MRTD scheme as the weighted mean of the conventional FDTD method. Taking $\mathbf{V}_{k}^{n+1}$ as an example, for $k=1,2, \ldots, L_{S}-1$, decomposing the iterative equation of $\mathbf{V}_{k}^{n+1}$ as (19a), (19b), (19c), and (19d), each subequation can be written as

$$
\begin{aligned}
& (2 i+1) a(i) \mathbf{V}_{k}^{n+1}=\mathbf{A}_{1}\left[(2 i+1) a(i) \mathbf{A}_{2} \mathbf{V}_{k}^{n+1}\right. \\
& \left.-(2 i+1) a(i) \frac{\Delta t}{(2 i+1) \Delta z}\left(\mathbf{I}_{k+i+1 / 2}^{n+1 / 2}-\mathbf{I}_{k-i-1 / 2}^{n+1 / 2}\right)\right]
\end{aligned}
$$

for $k=1,2, \ldots, L_{S}-1$.

Notice that if $k>i$, there does not exist a term exceeding the index range in subequation (38). That means the first $k$ terms of the subequations in (38) do not contain the exceeding index range terms, while the terms exceeding the index range all appear in the last $L_{S}-k$ subequations. By viewing MRTD schemes as the weighted mean of the conventional FDTD method, the last $L_{S}-k$ subequations are suitable for forming the iterative equations in MRTD schemes. Using the part summation to approximate the whole summation, we update the iterative equation of $\mathbf{V}_{k}^{n+1}$ by using the first $k$ subequations. The iterative equation becomes

$$
\begin{gathered}
\mathbf{V}_{k}^{n+1}=\mathbf{A}_{1}\left\{\mathbf{A}_{2} \mathbf{V}_{k}^{n}-\left[\sum_{i=0}^{k-1}(2 i+1) a(i)\right]^{-1} \frac{\Delta t}{\Delta z} \sum_{i=0}^{k-1} a(i)\right. \\
\left.\cdot\left(\mathbf{I}_{k+i+1 / 2}^{n+1 / 2}-\mathbf{I}_{k-i-1 / 2}^{n+1 / 2}\right)\right\}
\end{gathered}
$$

for $k=1,2, \ldots, L_{S}-1$.

Using the same method, we obtain the iterative equations of voltages and currents close to the terminals. The voltage iterative equations close to the load are

$$
\begin{gathered}
\mathbf{V}_{k}^{n+1}=\mathbf{A}_{1}\left\{\mathbf{A}_{2} \mathbf{V}_{k}^{n}-\left[\sum_{i=0}^{\mathrm{NDZ}-k-1}(2 i+1) a(i)\right]^{-1} \frac{\Delta t}{\Delta z}\right. \\
\left.\cdot \sum_{i=0}^{\mathrm{NDZ}-k-1} a(i)\left(\mathbf{I}_{k+i+1 / 2}^{n+1 / 2}-\mathbf{I}_{k-i-1 / 2}^{n+1 / 2}\right)\right\}
\end{gathered}
$$

for $k=\mathrm{NDZ}-L_{S}+1, \mathrm{NDZ}-L_{S}+2, \ldots, \mathrm{NDZ}-1$.

The current iterative equations close to the source are

$$
\begin{gathered}
\mathbf{I}_{k+1 / 2}^{n+1 / 2}=\mathbf{B}_{1}\left\{\mathbf{B}_{2} \mathbf{I}_{k+1 / 2}^{n-1 / 2}-\left[\sum_{i=0}^{k}(2 i+1) a(i)\right]^{-1} \frac{\Delta t}{\Delta z}\right. \\
\left.\cdot \sum_{i=0}^{k} a(i)\left(\mathbf{V}_{k+i+1}^{n}-\mathbf{V}_{k-i}^{n}\right)\right\}
\end{gathered}
$$

for $k=0,1, \ldots, L_{S}-2$.
The current iterative equations close to the load are

$$
\begin{aligned}
& \mathbf{I}_{k+1 / 2}^{n+1 / 2}=\mathbf{B}_{1}\left\{\mathbf{B}_{2} \mathbf{I}_{k+1 / 2}^{n-1 / 2}\right. \\
&- {\left[\sum_{i=0}^{\mathrm{NDZ}-k-1}(2 i+1) a(i)\right]^{-1} \frac{\Delta t}{\Delta z} } \\
&\left.\cdot \sum_{i=0}^{\mathrm{NDZ}-k-1} a(i)\left(\mathbf{V}_{k+i+1}^{n}-\mathbf{V}_{k-i}^{n}\right)\right\}
\end{aligned}
$$

for $k=\mathrm{NDZ}-L_{S}+1, \mathrm{NDZ}-L_{S}+2, \ldots, \mathrm{NDZ}-1$.

\section{Numerical Results}

4.1. Ribbon Cables. In this section, we consider a three-wire ribbon cable as shown in Figure 2. The total length of the line is $2 \mathrm{~m}$. One wire is chosen as the reference conductor and noted as Wire 0 , and other wires are noted as Wire 1 and Wire 2 , respectively. The source is located at the left side of Wire 2 , and the source resistance is $50 \Omega$. The left side of Wire 1 is a $50 \Omega$ resistance. The right side of Wire 1 and Wire 2 are both resistances with $50 \Omega$, and this model comes from [2], as shown in Figure 2(a).

The cross section of the three-wire ribbon cable is shown in Figure 2(b). The center-to-center separation of the wires is $d=50$ mils $\left(1 \mathrm{mil}=2.54 \times 10^{-5} \mathrm{~m}\right)$. The wires are identical and are composed of wires with radius of $r_{w}=7.5$ mils and polyvinyl chloride (PVC) insulations of thickness $t=$ 10 mils and relative dielectric constant $\varepsilon_{r}=3.5$. Following the method in [2], the per-unit-length inductance matrix $\mathbf{L}$ and capacitance matrix $\mathbf{C}$ are computed as

$$
\begin{aligned}
& \mathbf{L}=\left[\begin{array}{ll}
0.7485 & 0.5077 \\
0.5077 & 1.0154
\end{array}\right] \mu \mathrm{H} / \mathrm{m} \\
& \mathbf{C}=\left[\begin{array}{cc}
37.432 & -18.716 \\
-18.716 & 24.982
\end{array}\right] \mathrm{pF} / \mathrm{m} .
\end{aligned}
$$

Using $\mathbf{L}$ and $\mathbf{C}$ matrices above we can compute the mode velocities of propagation. They are $v_{m 1}=2.324 \times 10^{8} \mathrm{~m} / \mathrm{s}$ and $v_{m 2}=2.5106 \times 10^{8} \mathrm{~m} / \mathrm{s}$ giving one-way time delays of the modes of $T_{D m 1}=8.606 \mathrm{~ns}$ and $T_{D m 2}=7.996 \mathrm{~ns}$. The characteristic impedances of the modes are $Z_{\mathrm{Cm} 1}=91.96 \Omega$ and $Z_{\mathrm{Cm} 2}=254.93 \Omega$.

We use a ramp function as the source shown in Figure 3, the rising time of the ramp function is $\tau_{r}=2 \mathrm{~ns}$, and the total time is $T=200 \mathrm{~ns}$. The amplitude is $1 \mathrm{~V}$ and the initial value of the ramp function is $0 \mathrm{~V}$. The bandwidth of the source is approximate to $\mathrm{BW}=1 / \tau_{r}=500 \mathrm{MHz}$, so the highest frequency of the incident wave is approximately equal to $500 \mathrm{MHz}$. Taking $1 / 10$ of the wavelength of the highest frequency of the incident wave as the segment length, the segment length $\Delta z$ is $4.468 \times 10^{-2} \mathrm{~m}$. This space step is often suitable for FDTD method, so we use this segment length for numerical computing first. Since we divide the line into NDZ 


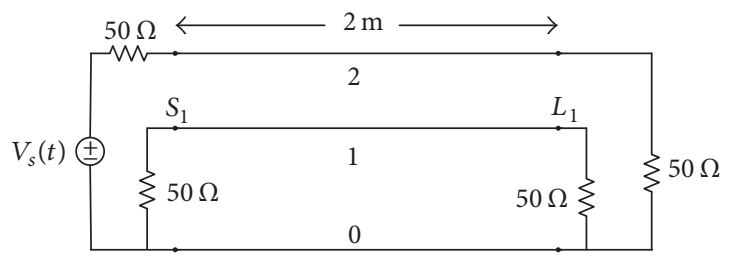

(a) Line dimensions and terminals

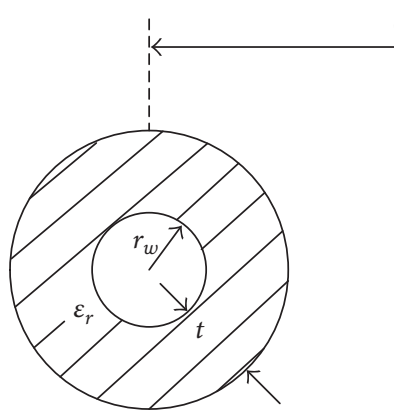

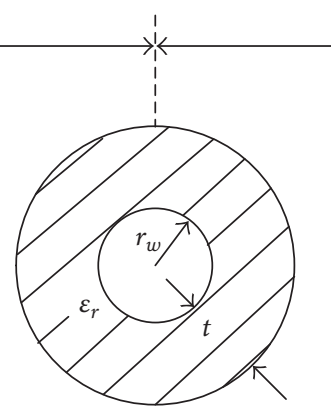

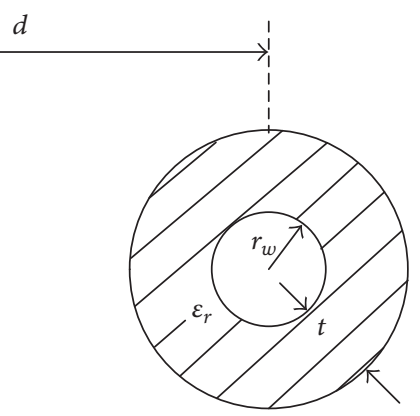

(b) Cross-sectional dimensions

Figure 2: A three-wire ribbon cable.

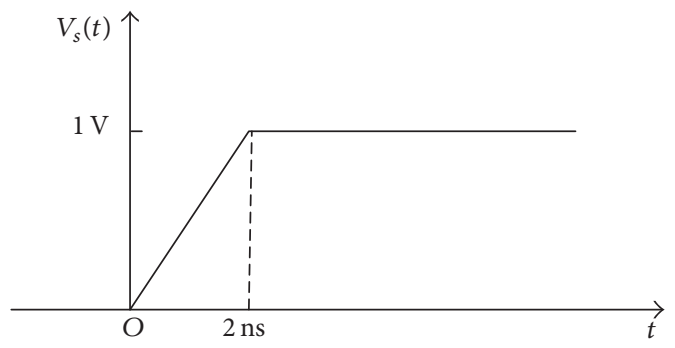

FIGURE 3: Waveform of the source voltage.

segments uniformly, the space discretization number is NDZ $=45$. For the stability of the algorithm, taking $q=0.5$, we can compute

$$
\mathrm{NDT}=\mathrm{NDZ} \times \frac{v \times T}{q \times L} .
$$

In Figures 4-12, we use Dbi-MRTD to represent the computed results of MRTD scheme by using $\mathrm{Db} i$ scaling functions as basis functions. Since the SPICE program can compute the response exactly for the lossless lines and the FDTD method has been proved to be an effective algorithm for the time-domain response, we compare the computed results of MRTD scheme with SPICE and FDTD methods to validate the effectiveness of the MRTD schemes.

Figure 4 shows the time-domain response of the leftend crosstalk of the ribbon cable by SPICE program, FDTD, and Db2-MRTD. It indicates that Db2-MRTD does not agree with the computed results of the SPICE program and the FDTD method. The reason for this phenomenon is that the scaling function of the Db2-wavelet does not have a high enough vanishing moment. When we use Daubechies' scaling functions to expand the voltages and currents in the MTL

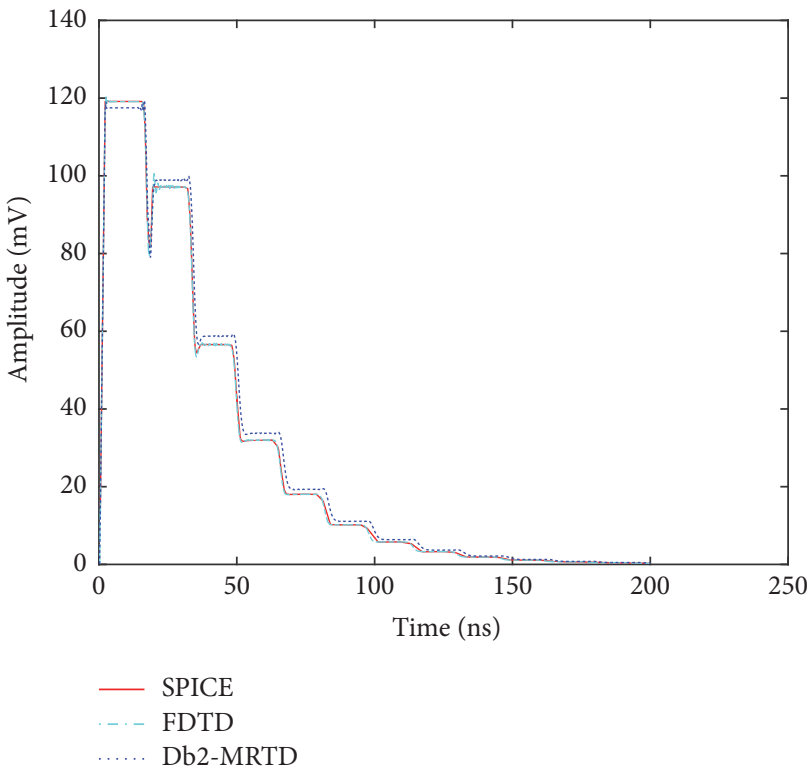

FIGURE 4: Comparison of the time-domain response of the left-end crosstalk of the ribbon cable by SPICE program, FDTD, and Db2MRTD. The space discretization number NDZ $=45$ and Courant number $q=0.5$.

equations, the vanishing moment decides the accuracy of the approximation. When using scaling functions with low vanishing moments as basis functions to approximate voltages and currents, such as Db2-wavelet's scaling functions, the approximation may introduce a larger error, while when using the scaling functions with high vanishing moments as basis functions, the approximation can be more accurate. 


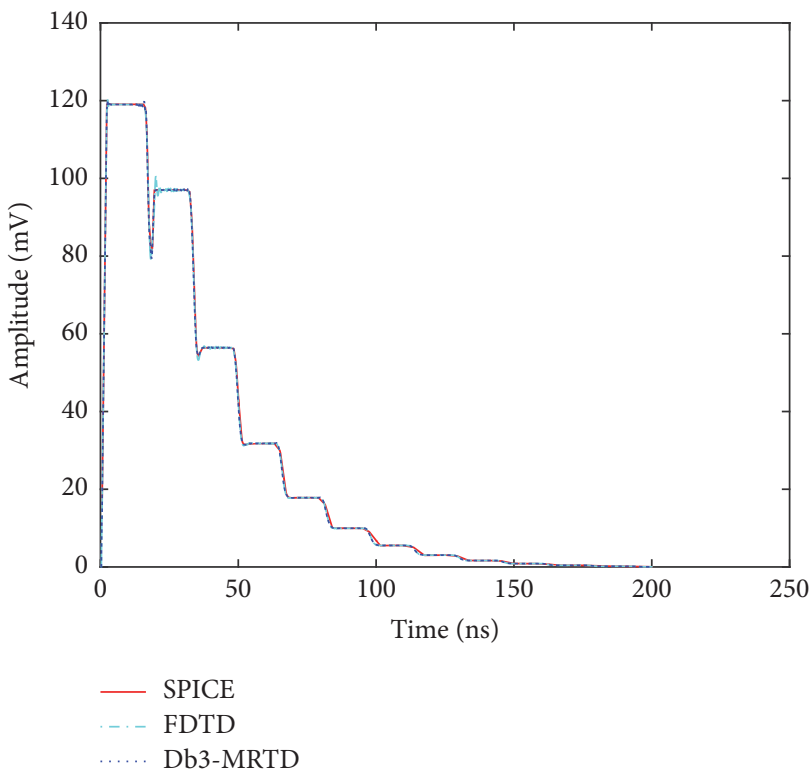

(a)

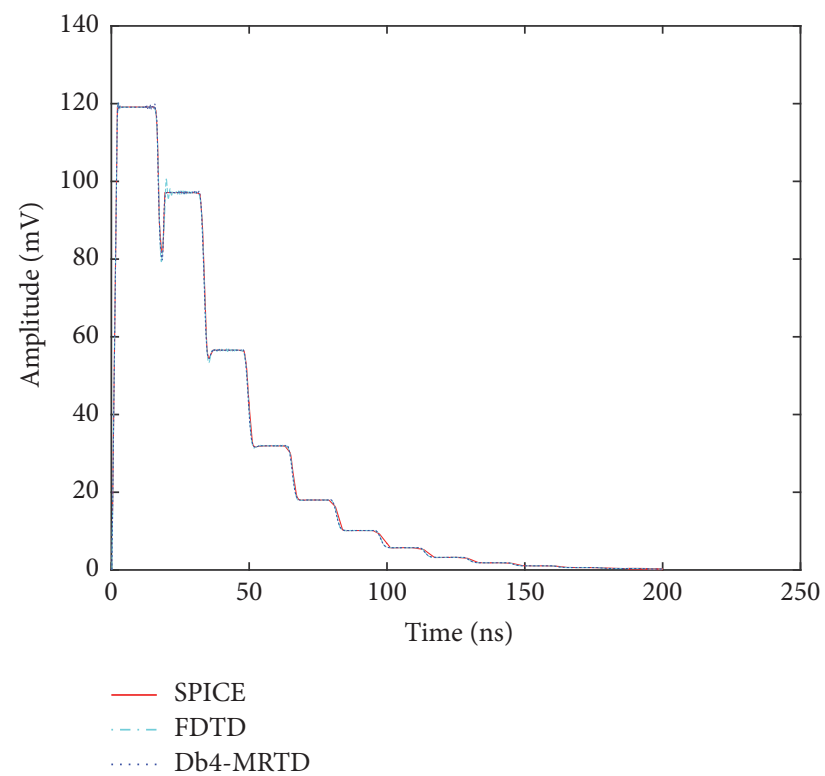

(b)

FIGURE 5: Comparison of the time-domain response of the left-end crosstalk of the ribbon cable by SPICE program, FDTD, and MRTD: (a) for Db3-MRTD and (b) for Db4-MRTD. The space discretization number NDZ $=45$ and Courant number $q=0.5$.

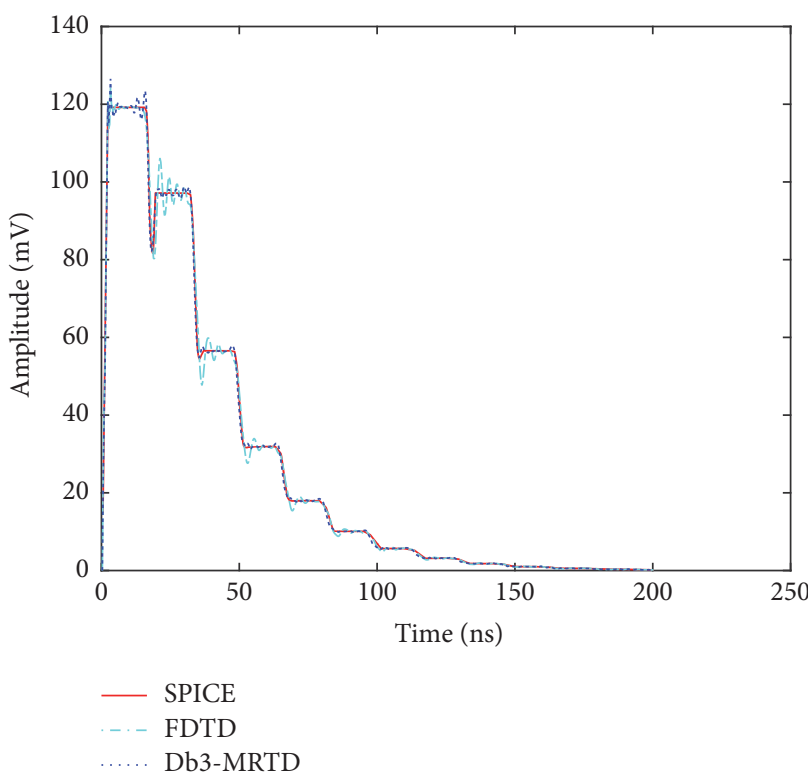

(a)

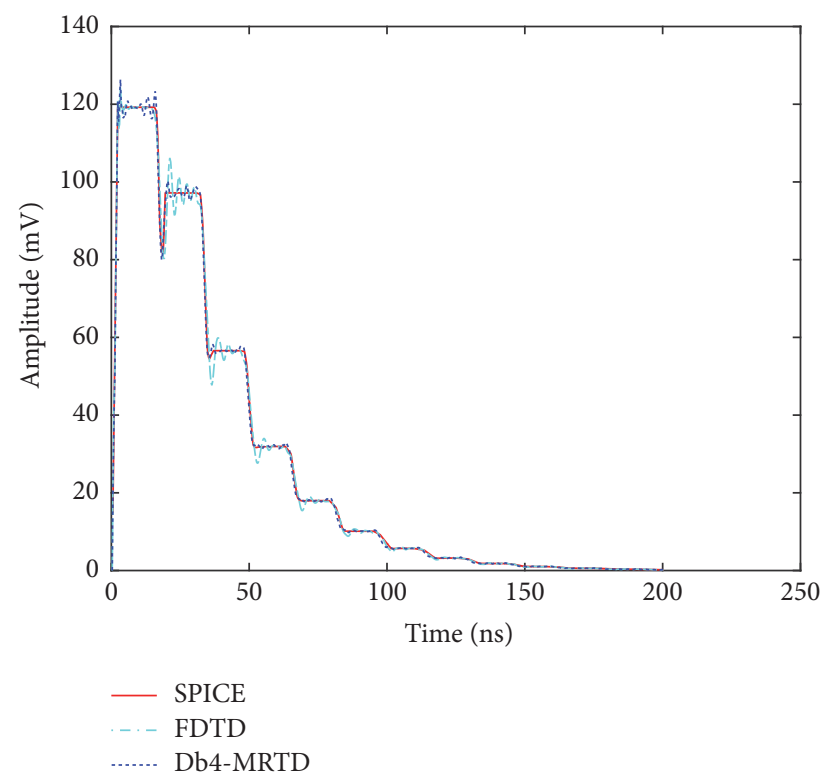

(b)

FIGURE 6: Comparison of the time-domain response of the left-end crosstalk of the ribbon cable by SPICE program, FDTD, and MRTD: (a) for Db3-MRTD and (b) for Db4-MRTD. The space discretization number NDZ $=12$ and Courant number $q=0.5$.

Figure 5 shows computed results of Db3-MRTD and Db4-MRTD. Both the two figures show the corresponding computing results by SPICE program, FDTD method, and MRTD schemes. The results of FDTD method show some oscillation on the edge of transition, while the computing results of Db3-MRTD and Db4-MRTD schemes show a better solution on the edge of transition.
To validate the advantage of the MRTD schemes in space sampling, we use a coarse space interval to compute the terminal response. Taking the segment length $\Delta z$ to be $\Delta z=$ $\lambda / 4$, the discretization will become $\mathrm{NDZ}=12$.

Figure 6 shows that both the computed results of FDTD method and MRTD schemes exhibit some oscillation on the edge of transition with the coarse space interval. The 


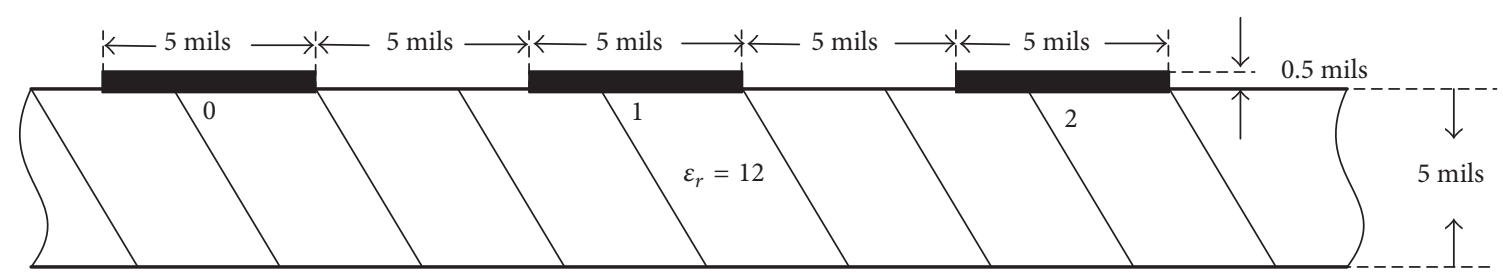

(a) Cross-sectional dimensions

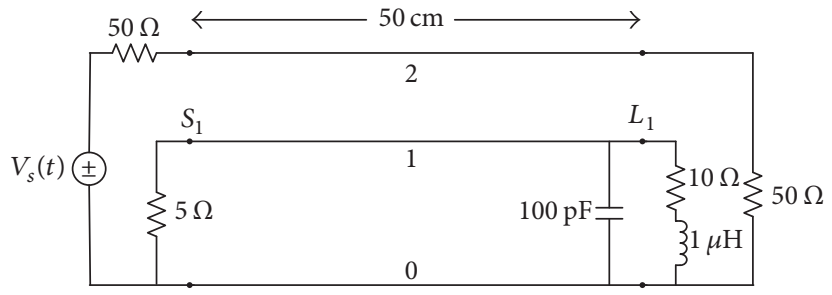

(b) Line dimensions and terminals

Figure 7: A printed circuit board.

TABle 2: Runtime for different schemes with different space discretization numbers.

\begin{tabular}{lcc}
\hline Different schemes & $\begin{array}{c}\text { Runtime for NDZ }= \\
12\end{array}$ & $\begin{array}{c}\text { Runtime for NDZ }= \\
45\end{array}$ \\
\hline FDTD & $0.0239 \mathrm{~s}$ & $0.1085 \mathrm{~s}$ \\
Db3-MRTD & $0.1146 \mathrm{~s}$ & $0.4693 \mathrm{~s}$ \\
Db4-MRTD & $0.1855 \mathrm{~s}$ & $0.7688 \mathrm{~s}$ \\
\hline
\end{tabular}

oscillation for the MRTD schemes appears almost at the first "stage" with a smaller amplitude; however, the oscillation for the FDTD method appears at several "stages" of the computed results with a larger amplitude. This indicates that we can use the MRTD schemes to obtain more accurate results than the conventional FDTD method when using the same grid.

Table 2 shows the runtime for different schemes with different space discretization numbers. The numerical results in Table 2 show that the runtime of Db3-MRTD is almost 5 times that of FDTD method, and the runtime of Db4MRTD is almost 7 times that of FDTD method. Since the effective support sizes of Db3's and Db5's scaling functions are 5 and 7 as shown in Table 1, the times of the runtime between MRTD and FDTD are corresponding to the size of the effective support of the basis functions. These results agree with the analysis in Section 3.

The numerical results in Figures 5 and 6 and Table 2 show that the MRTD schemes need more time to obtain more stable results when using the same space step with the conventional FDTD method. This indicates that the MRTD schemes allow obtaining more accurate results by sacrificing the computing efficiency.

4.2. Printed Circuit Boards. In this section, we compute the terminal response for a lossy printed circuit board. Three conductors of rectangular cross section of width $w=5$ mils and thickness $t=0.5$ mils are separated by $s=5$ mils and placed on one side of a silicon substrate having $\varepsilon_{r}=12$ and thickness $h=5$ mils [13], as shown in Figure 7(a), noting the reference as Wire 0 , and the other two lines are noted as Wire 1 and Wire 2.

Following the method in [2], the per-unit-length inductance and capacitance matrix are computed as

$$
\begin{aligned}
& \mathbf{L}=\left[\begin{array}{cc}
0.805756 & 0.538771 \\
0.538771 & 1.07754
\end{array}\right] \mu \mathrm{H} / \mathrm{m} \\
& \mathbf{C}=\left[\begin{array}{cc}
117.791 & -58.8956 \\
-58.8956 & 71.8544
\end{array}\right] \mathrm{pF} / \mathrm{m} .
\end{aligned}
$$

These give mode velocities in the lossless case of $v_{m 1}=$ $1.25809 \times 10^{8} \mathrm{~m} / \mathrm{s}$ and $v_{m 2}=1.47934 \times 10^{8} \mathrm{~m} / \mathrm{s}$.

Ignoring the dielectric loss, we can obtain $\mathbf{G}=\mathbf{0}$. The per-unit-length DC resistance of each conductor is computed as $r_{\mathrm{dc}}=1 /(\sigma w t)=10.6897 \Omega / \mathrm{m}$; just considering the $\mathrm{DC}$ resistance, we obtain

$$
\mathbf{R}=\left[\begin{array}{cc}
10.7897 & 0 \\
0 & 10.7897
\end{array}\right] \Omega / \mathrm{m}
$$

The total length of the line is $50 \mathrm{~cm}$ and the line is terminated with networks as shown in Figure 7(b). These networks at the source and load can be described by state 
variables as mentioned in Section 3. The state variables in (24a) and (24b) and (25a) and (25b) are listed below as [13]

$$
\begin{aligned}
& \mathbf{X}_{S}(t)=\mathbf{0}, \\
& \mathbf{M}_{S}=\mathbf{0} \text {, } \\
& \mathbf{N}_{1}=\mathbf{0} \text {, } \\
& \mathbf{N}_{S}=\mathbf{0} \text {, } \\
& \mathbf{O}_{S}=\mathbf{0} \text {, } \\
& \mathbf{P}_{1}=\left[\begin{array}{cc}
\frac{1}{R_{N E}} & 0 \\
0 & \frac{1}{R_{S}}
\end{array}\right], \\
& \mathbf{P}_{S}=\left[\begin{array}{cc}
\frac{1}{R_{N E}} & 0 \\
0 & \frac{1}{R_{S}}
\end{array}\right] \text {, } \\
& \mathbf{Q}_{1}=\mathbf{0} \text {, } \\
& \mathbf{Q}_{S}=\mathbf{0} \text {, } \\
& \mathbf{S}_{S}(t)=\left[\begin{array}{c}
0 \\
V_{S}(t)
\end{array}\right] \text {, } \\
& \mathbf{X}_{L}(t)=\left[\begin{array}{c}
i_{L}(t) \\
0
\end{array}\right] \text {, } \\
& \mathbf{M}_{L}=\left[\begin{array}{c}
-\frac{L}{R} \\
0
\end{array}\right] \text {, } \\
& \mathbf{N}_{\mathrm{NDZ}}=\left[\begin{array}{c}
\frac{1}{L} \\
0
\end{array}\right] \text {, } \\
& \mathbf{N}_{L}=\mathbf{0} \text {, } \\
& \mathbf{O}_{L}=\left[\begin{array}{l}
1 \\
0
\end{array}\right], \\
& \mathbf{P}_{\mathrm{NDZ}}=\left[\begin{array}{cc}
0 & 0 \\
0 & \frac{1}{R_{L}}
\end{array}\right], \\
& \mathbf{P}_{L}=\mathbf{0} \text {, } \\
& \mathbf{Q}_{\mathrm{NDZ}}=\left[\begin{array}{ll}
C & 0 \\
0 & 0
\end{array}\right] \text {, } \\
& \mathbf{Q}_{L}=\mathbf{0},
\end{aligned}
$$

where $C=100 \mathrm{pF}, L=1 \mu \mathrm{H}, R=10 \Omega, R_{S}=50 \Omega, R_{L}=$ $50 \Omega$, and $R_{N E}=5 \Omega$ and $i_{L}(t)$ is the inductor current.

The source is a trapezoidal pulse shown in Figure 8, and the amplitude is $1 \mathrm{~V}$. The rise time/fall time of the pulse

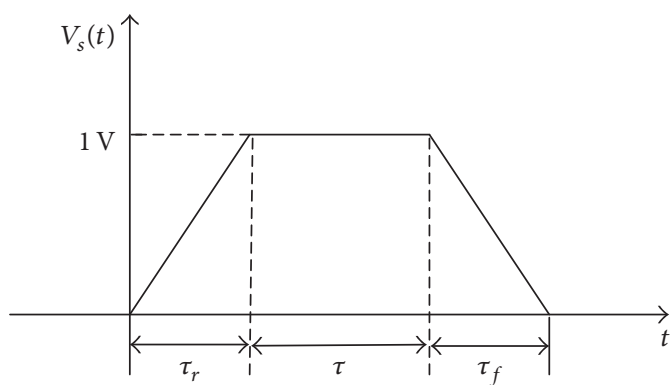

Figure 8: Waveform of a trapezoid pulse.

TABLE 3: Runtime for different schemes with different space discretization numbers.

\begin{tabular}{lccc}
\hline Different schemes & $\begin{array}{c}\text { Runtime for } \\
\text { NDZ }=199\end{array}$ & $\begin{array}{c}\text { Runtime for } \\
\text { NDZ }=398\end{array}$ & $\begin{array}{c}\text { Runtime for } \\
\text { NDZ =795 }\end{array}$ \\
\hline FDTD & $0.2060 \mathrm{~s}$ & $0.5999 \mathrm{~s}$ & $2.0181 \mathrm{~s}$ \\
Db3-MRTD & $0.9581 \mathrm{~s}$ & $2.7241 \mathrm{~s}$ & $8.7192 \mathrm{~s}$ \\
Db4-MRTD & $1.2724 \mathrm{~s}$ & $3.6920 \mathrm{~s}$ & $11.9143 \mathrm{~s}$ \\
\hline
\end{tabular}

are $\tau_{r}=\tau_{f}=100 \mathrm{ps}$ and pulse width is $\tau=500 \mathrm{ps}$. The total computing time is $40 \mathrm{~ns}$. The bandwidth of the input is approximately equal to $1 / \tau_{r}=10 \mathrm{GHz}$, taking the segment length as $\Delta z=\lambda / 10$. Using the small mode velocity $v_{m 1}$ at $10 \mathrm{GHz}$, we can get $\mathrm{NDZ}=398$. Choosing the Courant number $q$ satisfying the stability condition, the time discretization number NDT can be obtained from (44).

Figures 9(a) and 9(b) show terminal voltages of Wire 1 at the source and load by the FDTD method and the MRTD schemes. Since Db2-MRTD scheme is not an effective algorithm for MTL equations, we will just present the computed results of Db3-MRTD and Db4-MRTD. Both Figures 9(a) and 9 (b) show the agreement of these two kinds of method. It can be seen from Figure 9(a) that there exist more oscillations on the edge of each transition from the computed result of FDTD method, which means the FDTD method does not perform well with the rapid signals. On the other hand, the computed results also show that the MRTD schemes could lead to a more stable result for the rapid signals. And, for the gradually changing signals, both the two methods show good performance as shown in Figure 9(b).

To obtain a more accurate result, we could use a smaller space step and time step. Figures 10(a) and 10(b) show the computed results when we increase the space discretization number NDZ to 795. Both the figures show fewer oscillations on the edge of transition.

Figures 11(a) and 11(b) show the computed results when we decrease the space discretization step to $\Delta z=\lambda / 5$. Figure 11(a) also indicates that the MRTD schemes could obtain a more stable solution with a coarse space step compared to the conventional FDTD method.

Table 3 shows the runtime for Figures 9-11. The numerical results show that MRTD schemes need more runtime to obtain more stable results. The runtime of Db3-MRTD is 


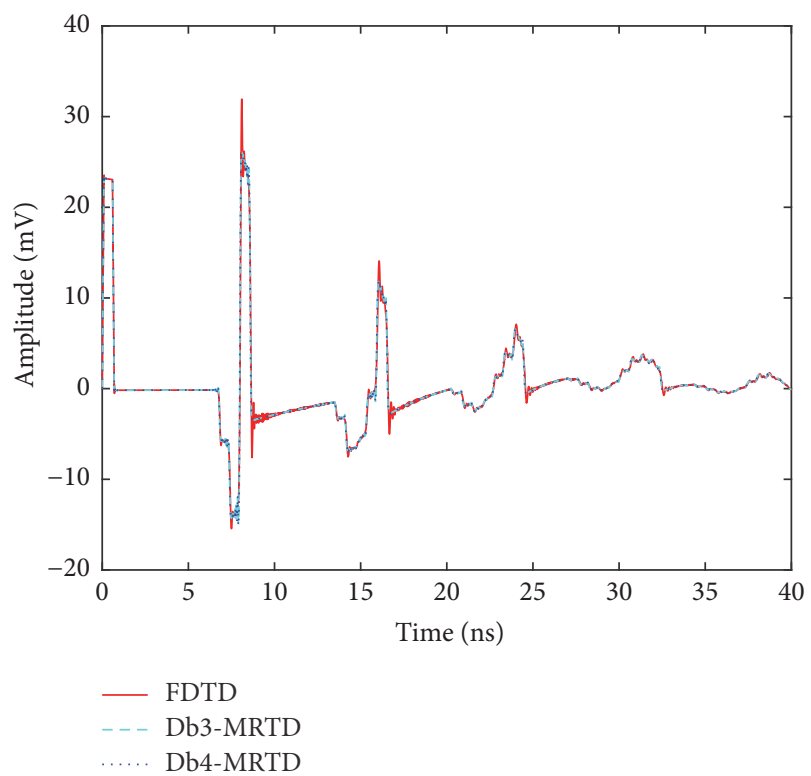

(a) Voltages at $S_{1}$

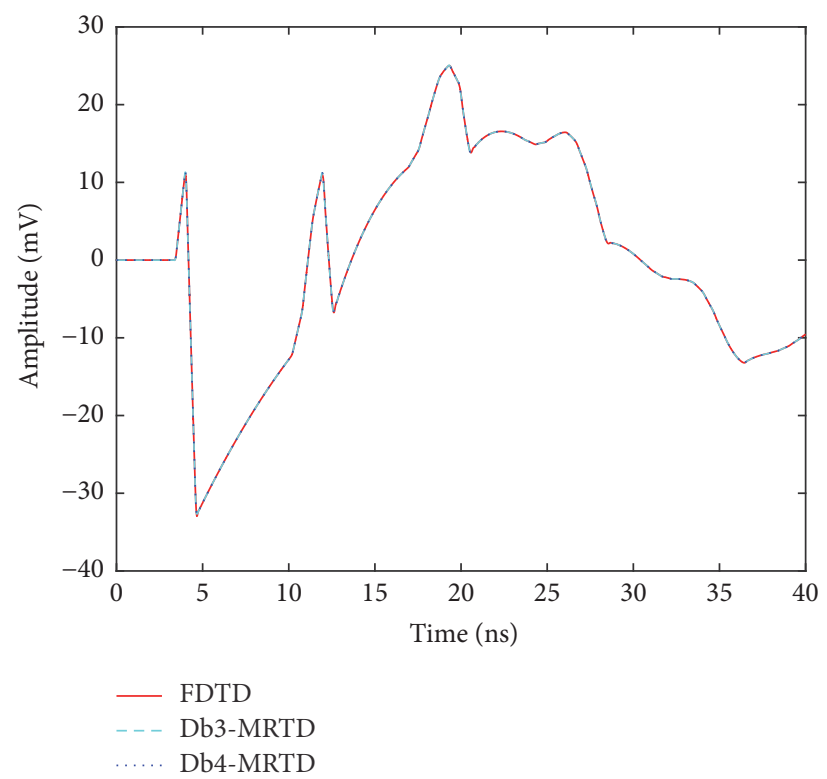

(b) Voltages at $L_{1}$

FIGURE 9: Computing results of terminal voltages for the lossy PCB by FDTD method and MRTD schemes. The space discretization number is $\mathrm{NDZ}=398$ and the Courant number is $q=0.5$.

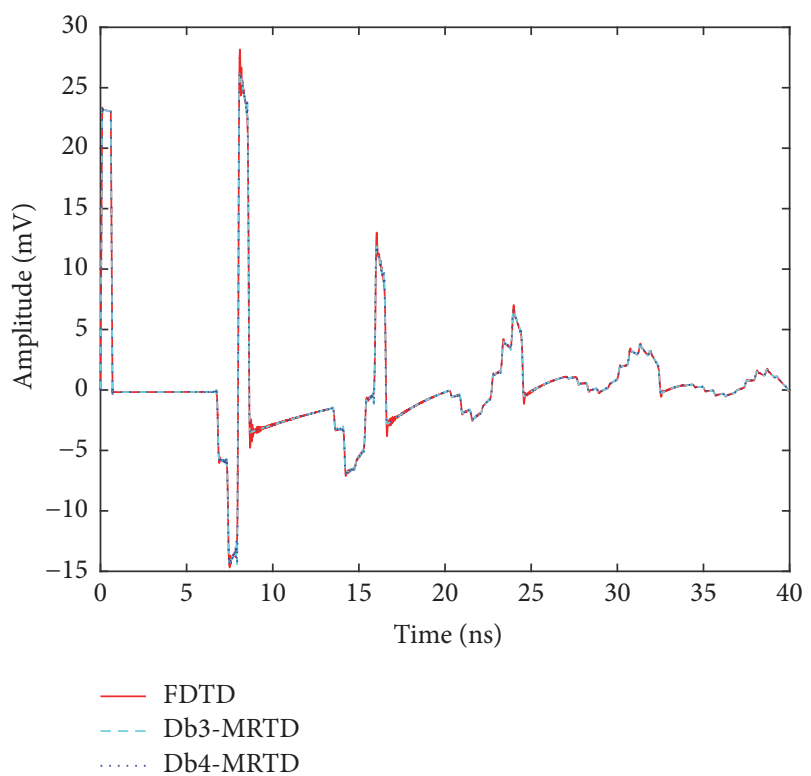

(a) Voltages at $S_{1}$

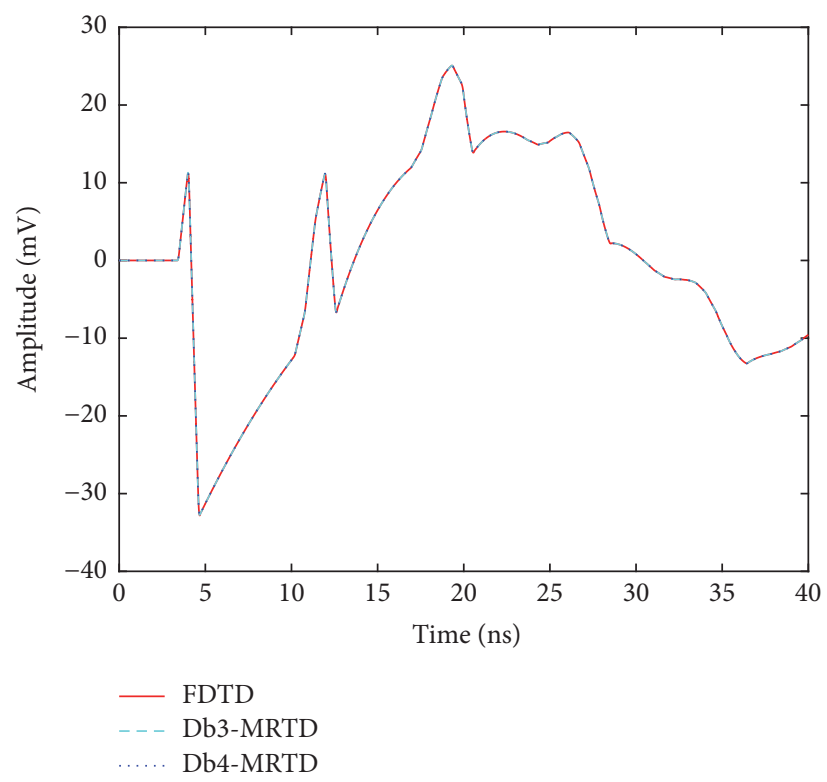

(b) Voltages at $L_{1}$

FIGURE 10: Computing results of terminal voltages for the lossy PCB by FDTD method and MRTD schemes. The space discretization number is $\mathrm{NDZ}=795$ and the Courant number is $q=0.5$.

almost 5 times that of FDTD method, and the result is corresponding to the analysis in Section 3. The runtime of Db4-MRTD is almost 6 times that of FDTD method, while the effective support size of the Db4's scaling functions is 7 . The runtime of Db4-MRTD is less than the effective support size of the Db4's scaling functions. The numerical results in Figures 9-11 and Table 3 also indicate that the MRTD schemes allow obtaining more accurate results by sacrificing the computing efficiency.

The oscillations may be caused by the oversampling of the time step, so we compute the terminal voltages by different Courant numbers. For FDTD, we take $q=1$ and, for MRTD schemes, we take the maximum Courant number for each MRTD scheme. Figure 12 shows the results. Since 


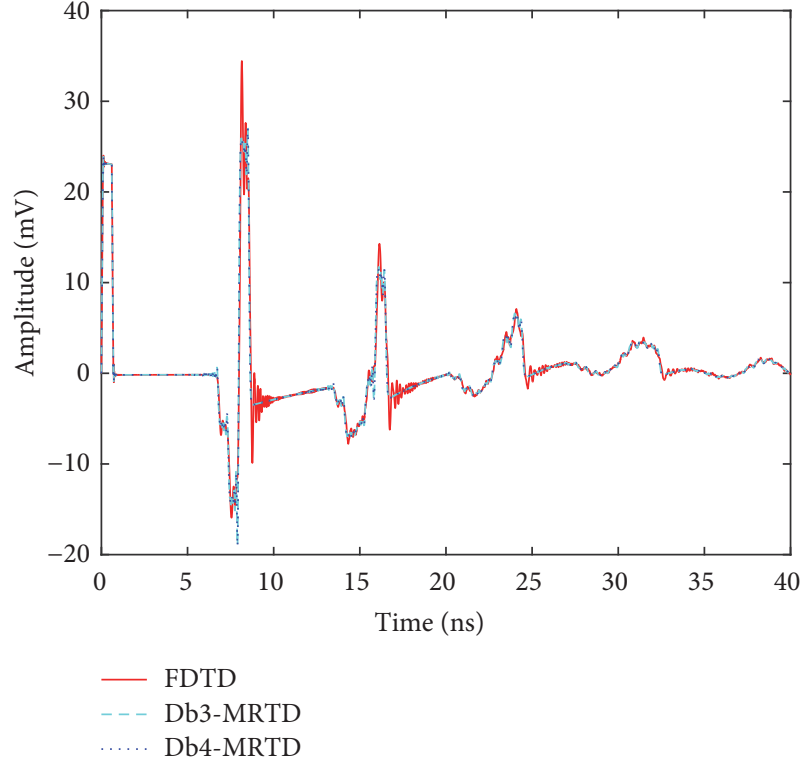

(a) Voltages at $S_{1}$

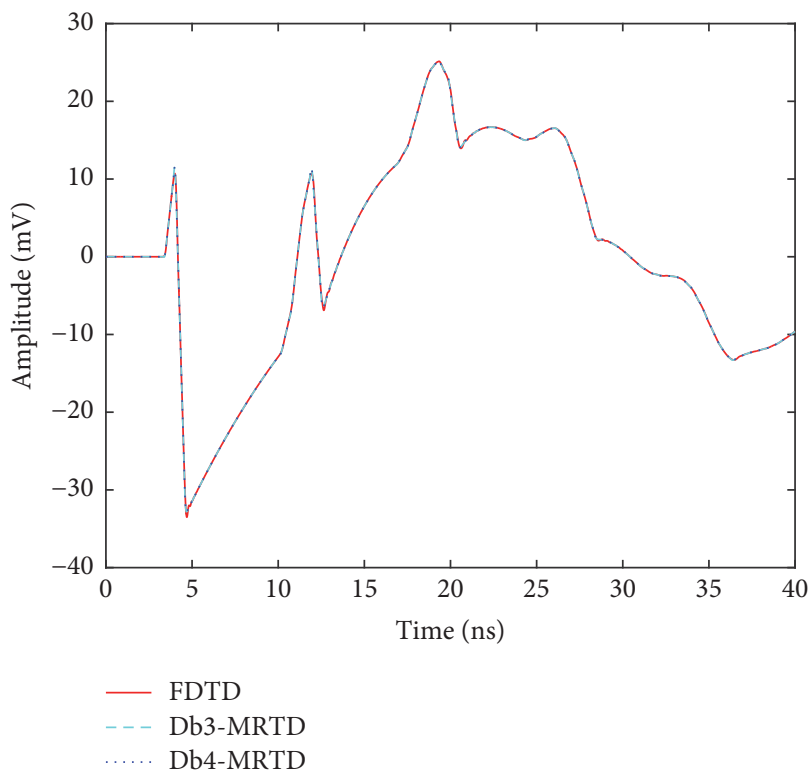

(b) Voltages at $L_{1}$

FIGURE 11: Computing results of terminal voltages for the lossy PCB by FDTD method and MRTD schemes. The space discretization number is $\mathrm{NDZ}=199$ and the Courant number is $q=0.5$.

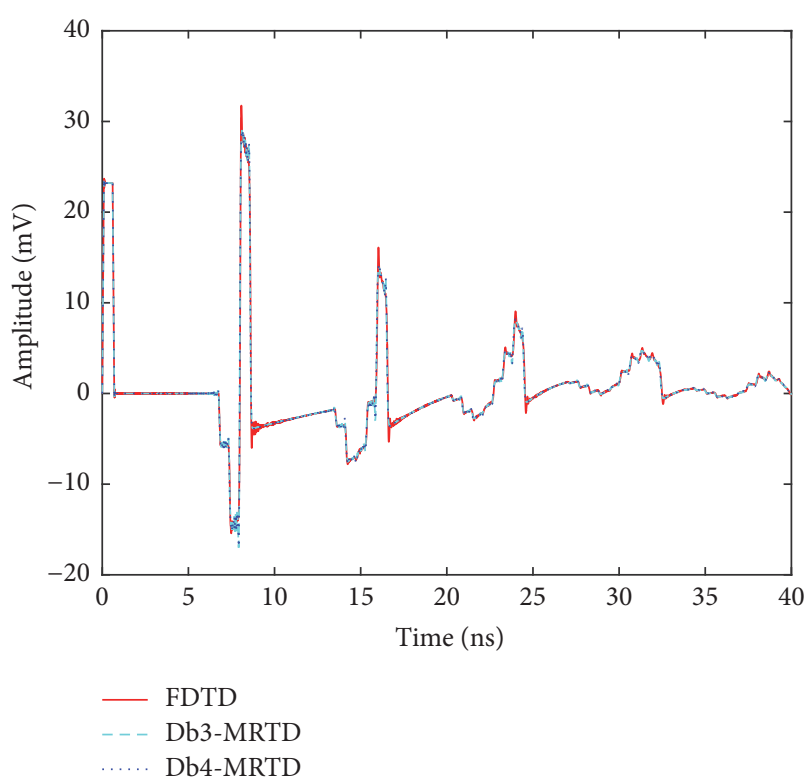

(a) Voltages at $S_{1}$

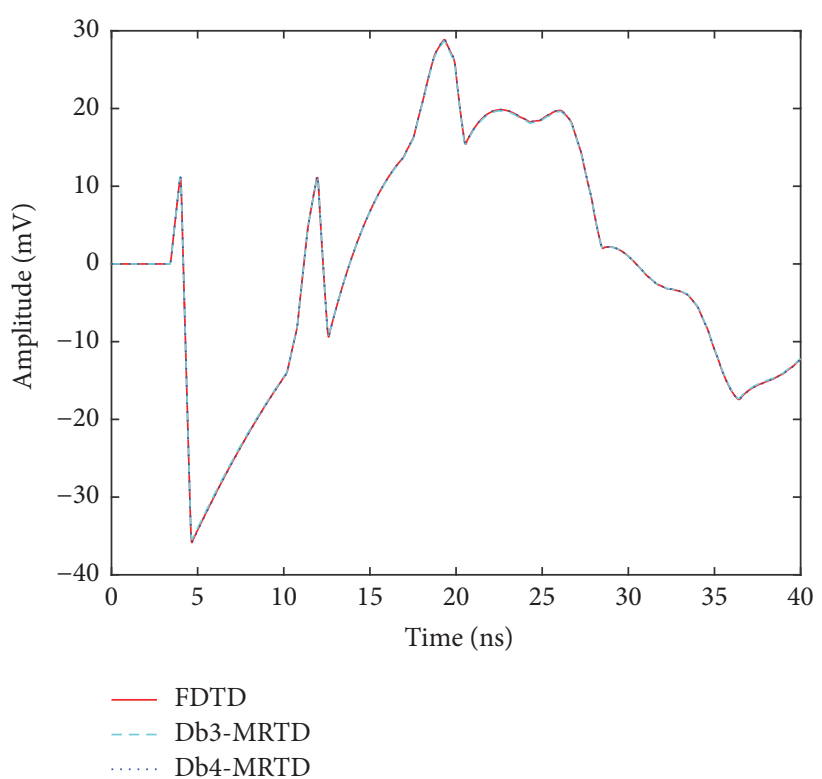

(b) Voltages at $L_{1}$

FIGURE 12: Computing results of terminal voltages for the lossy PCB by FDTD method and MRTD schemes. The space discretization number is NDZ $=398$, and the Courant numbers are $q_{\mathrm{FDTD}}=1, q_{\mathrm{D} b 3 \text {-MRTD }}=0.6844$, and $q_{\mathrm{D} b 4 \text {-MRTD }}=0.6585$ as shown in Table 1.

the MTLs have different mode velocity in each wire, the "magic step" for one dimension does not exist, and when we take $\Delta t=\Delta z / v_{\max }$ for the FDTD method, the oscillations do not disappear. Figure 12(a) shows that there exist more oscillations for the computing results of the FDTD methods with different Courant numbers. It means that the MRTD schemes are more stable than the FDTD methods for rapid signals.

\section{Conclusion}

In this paper, we derived the MRTD scheme for multiconductor transmission lines with linear terminations. By using state variant to describe the linear terminations, a method of incorporating the terminal constraints into the MRTD iterative equations is proposed to update the iterative equations which contain some terms whose indexes exceed 
the index range in MRTD schemes. Using different scaling functions as basis functions, the MRTD schemes are implemented for pure resistance terminations and linear network terminations including inductances and capacitances. The numerical results of MRTD schemes are compared to SPICE model and conventional FDTD, and the MRTD schemes are proved to be an effective algorithm for MTL equations and show a more stable performance with a coarse space step. However, the numerical results also show that the MRTD schemes need more time to obtain more stable results when compared to the conventional FDTD method. It indicates that the MRTD schemes allow obtaining more accurate results by sacrificing the computing efficiency.

\section{Conflicts of Interest}

The authors declare that they have no conflicts of interest.

\section{Acknowledgments}

This work was supported by the National Natural Science Foundation of China (no. 11271370).

\section{References}

[1] F. Rachidi and S. V. Tkachenko, Electromagnetic Field Interaction with Transmission Lines: From Classical Theory to HF Radiation Effects, vol. 1, WIT Press, Southampton, UK, 2008.

[2] C. R. Paul, Analysis of Multiconductor Transmission Lines, John Wiley \& Sons, 2nd edition, 2008.

[3] C. R. Paul, "A SPICE model for multiconductor transmission lines excited by an incident electromagnetic field," IEEE Transactions on Electromagnetic Compatibility, vol. 36, no. 4, pp. 342354,1994

[4] I. Maio, F. G. Canavero, and B. Dilecce, "Analysis of crosstalk and field coupling to lossy MTLs in a SPICE environment," IEEE Transactions on Electromagnetic Compatibility, vol. 38, no. 3, pp. 221-229, 1996.

[5] I. Erdin, A. Dounavis, R. Achar, and M. S. Nakhla, "A SPICE model for incident field coupling to lossy multiconductor transmission lines," IEEE Transactions on Electromagnetic Compatibility, vol. 43, no. 4, pp. 485-494, 2001.

[6] G. S. Shinh, N. M. Nakhla, R. Achar, M. S. Nakhla, A. Dounavis, and I. Erdin, "Fast transient analysis of incident field coupling to multiconductor transmission lines," IEEE Transactions on Electromagnetic Compatibility, vol. 48, no. 1, pp. 57-73, 2006.

[7] A. Dounavis, X. Li, M. S. Nakhla, and R. Achar, "Passive closedform transmission-line model for general-purpose circuit simulators," IEEE Transactions on Microwave Theory and Techniques, vol. 47, no. 12, pp. 2450-2459, 1999.

[8] A. Dounavis, R. Achar, and M. S. Nakhla, "Efficient passive circuit models for distributed networks with frequency-dependent parameters," IEEE Transactions on Advanced Packaging, vol. 23, no. 3, pp. 382-392, 2000.

[9] G. Antonini and G. Ferri, "A general class of passive macromodels for lossy multiconductor transmission lines," IEEE Transactions on Microwave Theory and Techniques, vol. 49, no. 10, pp. 1686-1696, 2001.

[10] G. Antonini and G. Ferri, "A new approach for closed-form transient analysis of multiconductor transmission lines," IEEE
Transactions on Electromagnetic Compatibility, vol. 46, no. 4, pp. 529-543, 2004.

[11] C. R. Paul, "Incorporation of terminal constraints in the FDTD analysis of transmission lines," IEEE Transactions on Electromagnetic Compatibility, vol. 36, no. 2, pp. 85-91, 1994.

[12] J. A. Roden, C. R. Paul, W. T. Smith, and S. D. Gedney, "Finitedifference, time-domain analysis of lossy transmission lines," IEEE Transactions on Electromagnetic Compatibility, vol. 38, no. 1, pp. 15-24, 1996.

[13] A. Orlandi and C. R. Paul, "FDTD analysis of lossy, multiconductor transmission lines terminated in arbitrary loads," IEEE Transactions on Electromagnetic Compatibility, vol. 38, no. 3, pp. 388-399, 1996.

[14] M. Krumpholz and L. P. B. Katehi, "MRTD: new time-domain schemes based on multiresolution analysis," IEEE Transactions on Microwave Theory and Techniques, vol. 44, no. 4, pp. 555-571, 1996.

[15] M. Fujii and W. J. R. Hoefer, "A three-dimensional haar-waveletbased multiresolution analysis similar to the FDTD methodderivation and application5," IEEE Transactions on Microwave Theory and Techniques, vol. 46, no. 12, pp. 2463-2475, 1998.

[16] Y. W. Cheong, Y. M. Lee, K. H. Ra, J. G. Kang, and C. C. Shin, "Wavelet-Galerkin scheme of time-dependent inhomogeneous electromagnetic problems," IEEE Microwave and Wireless Components Letters, vol. 9, no. 8, pp. 297-299, 1999.

[17] T. Dogaru and L. Carin, "Multiresolution time-domain using CDF biorthogonal wavelets," IEEE Transactions on Microwave Theory and Techniques, vol. 49, no. 5, pp. 902-912, 2001.

[18] X. Zhu, T. Dogaru, and L. Carin, "Analysis of the CDF biorthogonal MRTD method with application to PEC targets," IEEE Transactions on Microwave Theory and Techniques, vol. 51, no. 9, pp. 2015-2022, 2003.

[19] X. Wei, E. Li, and C. Liang, "A new MRTD scheme based on Coifman scaling functions for the solution of scattering problems," IEEE Microwave and Wireless Components Letters, vol. 12, no. 10, pp. 392-394, 2002.

[20] E. M. Tentzeris, R. L. Robertson, J. F. Harvey, and L. P. B. Katehi, "Stability and dispersion analysis of battle-lemariebased MRTD schemes," IEEE Transactions on Microwave Theory and Techniques, vol. 47, no. 7, pp. 1004-1013, 1999.

[21] S. Grivet-Talocia, "On the accuracy of Haar-based multiresolution time-domain schemes," IEEE Microwave and Wireless Components Letters, vol. 10, no. 10, pp. 397-399, 2000.

[22] M. Fujii and W. J. R. Hoefer, "Dispersion of time domain wavelet galerkin method based on Daubechies' compactly supported scaling functions with three and four vanishing moments," IEEE Microwave and Wireless Components Letters, vol. 10, no. 4, pp. 125-127, 2000.

[23] A. Alighanbari and C. D. Sarris, "Dispersion properties and applications of the Coifman scaling function based S-MRTD," IEEE Transactions on Antennas and Propagation, vol. 54, no. 8, pp. 2316-2325, 2006.

[24] K. L. Shlager and J. B. Schneider, "Comparison of the dispersion properties of several low-dispersion finite-difference time-domain algorithms," IEEE Transactions on Antennas and Propagation, vol. 51, no. 3, pp. 642-653, 2003.

[25] Z. Tong, L. Sun, Y. Li, and J. Luo, "Multiresolution time-domain scheme for terminal response of two-conductor transmission lines," Mathematical Problems in Engineering, vol. 2016, Article ID 8045749, 15 pages, 2016 . 
[26] L. O. Chua and P. M. Lin, Computer Aided Analysis of Electronic Circuits: Algorithms and Computational Techniques, PrenticeHall, 1975.

[27] B. J. Leon, Lumped Systems, Halt, finehart, and Winston, 1968.

[28] W. Sweldens and R. Piessens, "Wavelet sampling techniques," in Proceedings of the Statistical Computing Section, pp. 20-29, American Statistical Association, 1993.

[29] I. Daubechies, Ten Lectures on Wavelets, SIAM Press, 1992.

[30] A. Taflove and S. C. Hagness, Computational Electrodynamics: The Finite-Difference Time-Domain Method, Artech House, 3rd edition, 2005. 


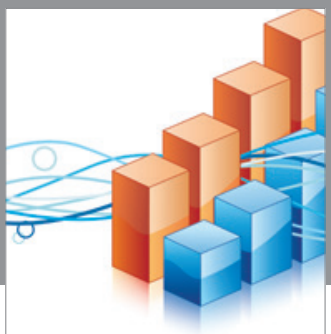

Advances in

Operations Research

vatem alat4

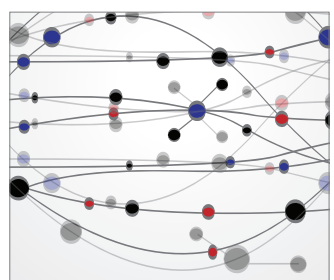

\section{The Scientific} World Journal
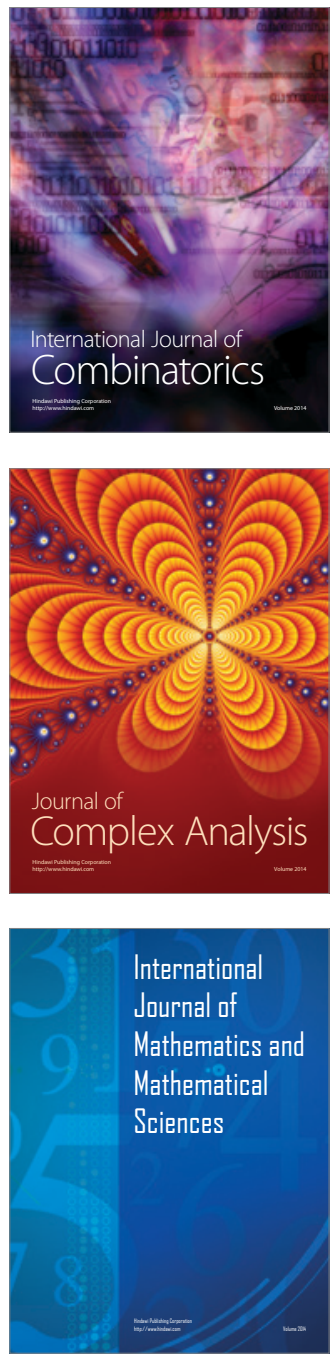
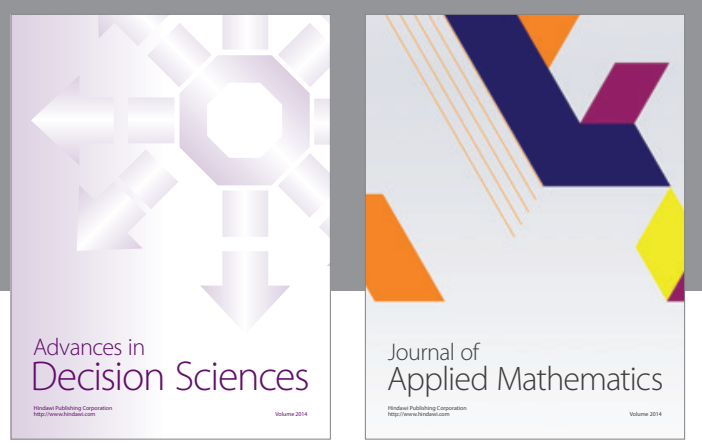

Algebra

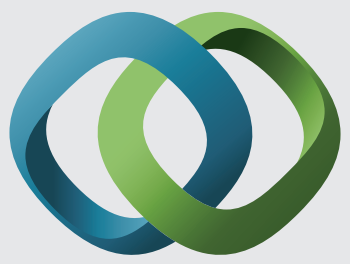

\section{Hindawi}

Submit your manuscripts at

https://www.hindawi.com
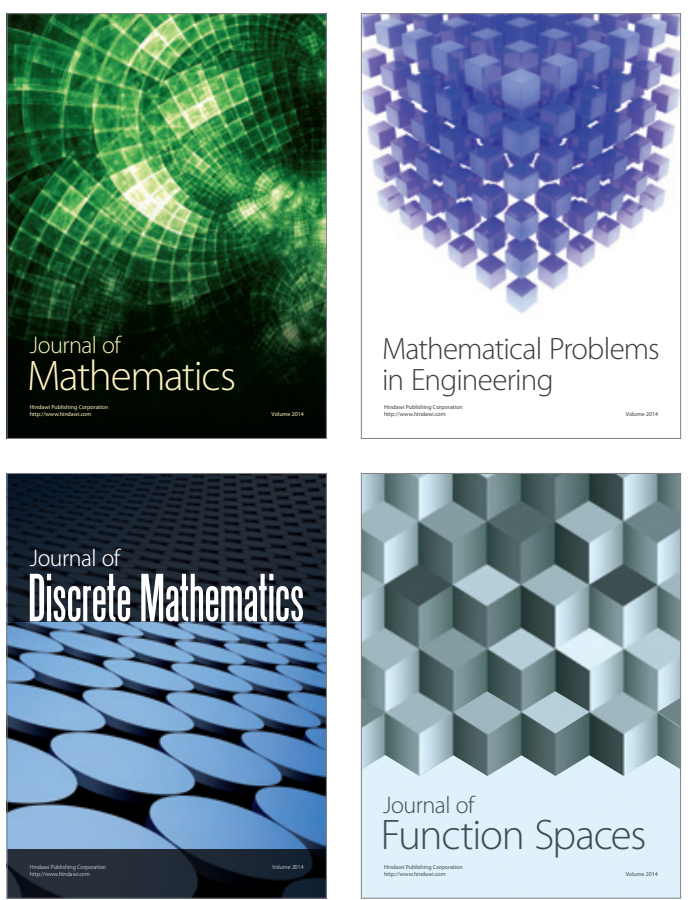

Mathematical Problems in Engineering
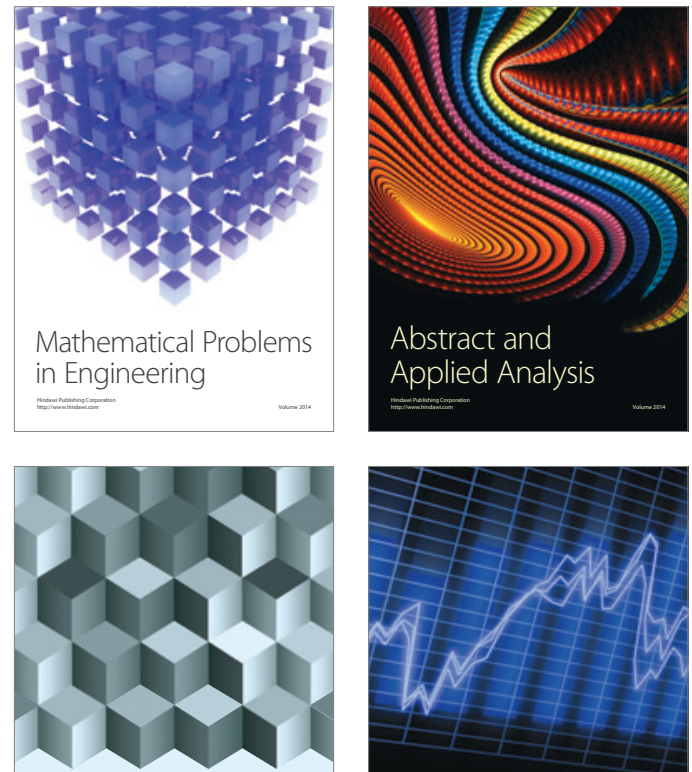

Journal of

Function Spaces

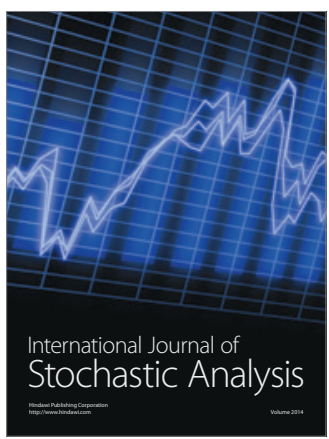

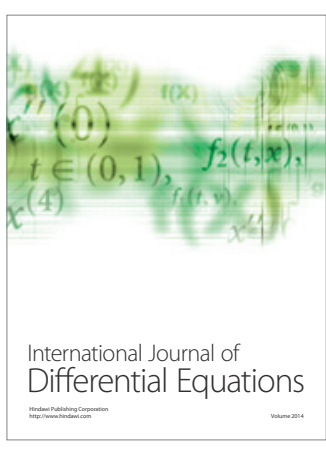
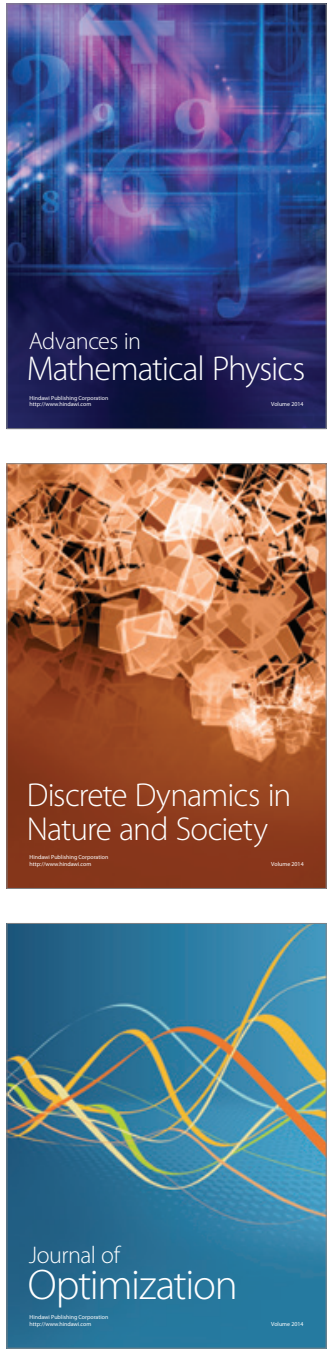